\title{
Closed-Loop Quantitative Verification of Rate-Adaptive Pacemakers
}

\author{
NICOLA PAOLETTI, University of Oxford, Department of Computer Science \\ ANDREA PATANĖ, University of Catania, Department of Mathematics and Computer Science \\ MARTA KWIATKOWSKA, University of Oxford, Department of Computer Science
}

\begin{abstract}
Rate-adaptive pacemakers are cardiac devices able to automatically adjust the pacing rate in patients with chronotropic incompetence, i.e., whose heart is unable to provide an adequate rate at increasing levels of physical, mental, or emotional activity. These devices work by processing data from physiological sensors in order to detect the patient's activity and update the pacing rate accordingly. Rate adaptation parameters depend on many patient-specific factors, and effective personalization of such treatments can only be achieved through extensive exercise testing, which is normally intolerable for a cardiac patient. In this work, we introduce a data-driven and model-based approach for the automated verification of rate-adaptive pacemakers and formal analysis of personalized treatments. To this purpose, we develop a novel dual-sensor pacemaker model where the adaptive rate is computed by blending information from an accelerometer, and a metabolic sensor based on the QT interval. Our approach enables personalization through the estimation of heart model parameters from patient data (electrocardiogram), and closed-loop analysis through the online generation of synthetic, model-based QT intervals and acceleration signals. In addition to personalization, we also support the derivation of models able to account for the varied characteristics of a virtual patient population, thus enabling safety verification of the device. To capture the probabilistic and nonlinear dynamics of the heart, we define a probabilistic extension of timed I/O automata with data and employ statistical model checking for quantitative verification of rate modulation. We evaluate our rate-adaptive pacemaker design on three subjects and a pool of virtual patients, demonstrating the potential of our approach to provide rigorous, quantitative insights into the closed-loop behavior of the device under different exercise levels and heart conditions.
\end{abstract}

CCS Concepts: • General and reference $\rightarrow$ Verification; • Computer systems organization $\rightarrow$ Embedded and cyber-physical systems; • Applied computing $\rightarrow$ Life and medical sciences;

Additional Key Words and Phrases: Quantitative verification, PTIOA

\section{ACM Reference format:}

Nicola Paoletti, Andrea Patanè, and Marta Kwiatkowska. 2018. Closed-Loop Quantitative Verification of RateAdaptive Pacemakers. ACM Trans. Cyber-Phys. Syst. 2, 4, Article 33 (August 2018), 31 pages.

https://doi.org/10.1145/3152767

This work is supported by the ERC AdG VERIWARE, the ERC PoC VERIPACE, and AFFECTech ITN. Nicola Paoletti is partially supported by the NSF Grant CNS-1446832. Andrea Patanè carried out the research during his internship at the University of Oxford funded by VERIWARE and VERIPACE projects.

Authors' addresses: N. Paoletti (Current address), Department of Computer Science, Royal Holloway, University of London, Egham Hill, Egham TW20 0EX, UK; email: nclpltt@gmail.com; A. Patanè and M. Kwiatkowska, Department of Computer Science, University of Oxford, Wolfson Building, Parks Road, Oxford, OX1 3QD, UK; emails: \{andrea.patane, Marta.Kwiatkowska\}@cs.ox.ac.uk.

Permission to make digital or hard copies of all or part of this work for personal or classroom use is granted without fee provided that copies are not made or distributed for profit or commercial advantage and that copies bear this notice and the full citation on the first page. Copyrights for components of this work owned by others than ACM must be honored. Abstracting with credit is permitted. To copy otherwise, or republish, to post on servers or to redistribute to lists, requires prior specific permission and/or a fee. Request permissions from permissions@acm.org.

(c) 2018 ACM 2378-962X/2018/08-ART33 $\$ 15.00$

https://doi.org/10.1145/3152767

ACM Transactions on Cyber-Physical Systems, Vol. 2, No. 4, Article 33. Publication date: August 2018. 


\section{INTRODUCTION}

Cardiac pacemakers are small, life-saving medical devices that stimulate the heart tissue through artificial electrical impulses in order to maintain an adequate heart rhythm in patients with cardiac conditions. Current pacemakers include mechanisms for rate adaptation, i.e., for automatically adjusting the frequency of electrical stimuli, or pacing rate, depending on the levels of physical, mental, or emotional stress of the patient. This feature is crucial to ensure a good quality of life for patients with chronotropic incompetence, i.e., whose heart is unable to provide by itself a rate commensurate with the ideal metabolic demand. Rate-adaptive pacemakers work by processing data from physiological sensors in order to detect the patient's activity and update the pacing rate accordingly (Dell'Orto et al. 2004).

Rate adaptation and device safety have been investigated in a number of clinical studies (see, e.g., Candinas et al. (1997), Lamas et al. (2007), and Abi-Samra et al. (2013)), but such studies require extensive testing under varying levels of physical activity, including maximal exercise tests (e.g., treadmill), which is often intolerable for cardiac patients, especially the elderly. Similarly, exercise testing is necessary for effective personalization of the treatment, given that rate adaptation parameters depend on many patient-specific factors such as age, lifestyle, and tolerance to rapid pacing.

An alternative approach put forward in this article is rigorous in silico analysis of the designs, while accounting for the specific electrophysiological characteristics of the patient and how these characteristics vary at different activity levels.

We introduce a data-driven, model-based approach for the automated, closed-loop verification of rate-adaptive pacemakers. We consider the $\mathrm{VVIR}^{1}$ pacemaker design, that is, a single-chamber pacemaker that senses and paces the (right) ventricle and supports rate adaptation. To this purpose, we develop a novel dual-sensor VVIR pacemaker model where the adaptive rate is computed by a so-called sensor blending algorithm that combines information coming from two sensors: an accelerometer, and a metabolic sensor based on the QT interval (QTI), i.e., the time needed for ventricle depolarization and repolarization. QTIs are extracted from the pacemaker electrogram, i.e., the electrical signal recorded by the electrode.

The proposed sensor blending algorithm exploits the strengths of both sensors: the accelerometer has a quick response to exercise but is inaccurate, while the QTI provides a slow but very accurate response. The QT sensor relies on the fact that physical and mental stress shortens the QTI. In particular, there is a natural, patient-specific relationship between QTI and heart rate (HR) in healthy subjects. The blending algorithm leverages this relationship through the estimation of regression functions between QTI and HR, used to predict an adequate pacing rate based on the QTI.

A key feature of our approach is that it supports rigorous analysis of patient-specific treatments through the estimation of personalized heart models from electrocardiogram (ECG) data (Barbot et al. 2015a). Importantly, our estimation method can also handle ECG data from multiple patients, by combining the parameters (i.e., probability distributions extracted from data) across all patients in a given population. In this way, we can estimate models able to fully capture the varied characteristics of the virtual patient population, thus enabling safety verification.

To account for the probabilistic nature of cardiac dynamics and ECG features detected from data, heart and pacemaker models are specified in a probabilistic extension of timed I/O automata with priorities and data (Kwiatkowska et al. 2015; Barbot et al. 2016). This formalism can represent networked systems with real-time constraints and discrete control actions, characteristic of the

${ }^{1}$ The code VVIR is based on the standard NASPE/BPEG nomenclature (Bernstein et al. 2002). 
pacemaker, as well as the probabilistic, hybrid, and nonlinear dynamics of the cardiac conduction system. For formal verification, we resort to statistical model checking (SMC) (Ballarini et al. 2015), an approximate verification technique based on the statistical inference of quantitative properties from a set of executions, well suited to our formalism for which no precise model checking method exists.

A crucial requirement for the analysis in silico of rate adaptation is closing the gap between the heart simulation and the physiological sensor data used by the pacemaker to update the pacing rate, in this way modeling the characteristic feedback interaction between the physiological signal used and the heart dynamics (Ellenbogen et al. 2016, ch. 5). To this aim, we introduce a closed-loop design for rate adaptation that builds on the online generation of model-based QTIs and synthetic accelerometer signals.

We evaluate our approach over four different heart model parameterizations, respectively describing three distinct virtual patients and a pool of 10 virtual patients, and employ SMC for quantitative verification in a variety of scenarios. These include the analysis of rate control under realistic exercise curves and clinical stress tests, and under increasing degrees of chronotropic incompetence (i.e., worsening heart conditions). Results demonstrate that our approach can provide rigorous and quantitative insights into the safety and performance of the device, enabling both personalized and population-level analysis of cardiac therapies, with the potential to drastically reduce the need for actual exercise testing with cardiac patients.

Article Structure. In the remainder of the introduction we discuss related work. In Section 2, we introduce our main modeling formalism. Statistical model checking is presented in Section 3. In Section 4, we introduce our closed-loop heart-pacemaker model, the virtual patients used for our analyses, and the methods for the processing and generation of physiological sensor data. In Section 5, we report simulation and verification results. Conclusions are given in Section 6.

\subsection{Related Work}

This article is a major extension of Kwiatkowska et al. (2014), which introduces the first formal and executable model of the VVIR pacemaker. In that work, the authors realize a single-sensor and open-loop design for rate adaptation, i.e., such that the pacemaker detects activity from static, offline physiological data and just from one sensor (the QT). In contrast, our work implements a dual-sensor and closed-loop design, which provides faster and more accurate activity detection through the combination of QT and accelerometer sensors, and uses dynamically generated physiological information. Compared to the open-loop design, in Section 5.2.2, we show that the closed-loop design can reproduce with superior accuracy phenomena of sensor-induced endlessloop tachycardia, triggered by the feedback interaction between the pacemaker, the heart, and the resulting physiological sensor data. Furthermore, in this work, we employ the probabilistic heart model of Barbot et al. (2015a) that supports personalization from patient data. This improves on Kwiatkowska et al. (2014), where the authors use the deterministic heart model of Ye et al. (2005), which, albeit providing more detailed action potential dynamics, does not support personalization.

Formal modeling and analysis of cardiac dynamics and devices is a very active field of research (see Macedo et al. (2008), Gomes and Oliveira (2009), Bartocci et al. (2009), Tuan et al. (2010), Grosu et al. (2011), Jiang et al. (2012), Pajic et al. (2012), Méry et al. (2014), Chen et al. (2014), Kwiatkowska et al. (2015), Barker et al. (2015), and Ai et al. (2018) for a nonexhaustive list of references). However, modeling of the rate-adaptive pacemaker has received limited attention so far. Besides Kwiatkowska et al. (2014) (discussed above), to the best of our knowledge, the only other work on rate adaptation is that of Méry and Singh (2009), where the authors consider a singlesensor accelerometer-based pacemaker, but only provide a high-level, nonexecutable specification 
of the rate modulation mechanism. Related research also includes the definition of algorithms for integrating signals from multiple physiological sensors (see, e.g., Shin et al. (2001) and Amigoni et al. (2006)).

A complementary technique is model-based testing (MBT), employed by Ai et al. (2016) for the validation of cardiac devices. Similarly to SMC, MBT relies on evaluating executions of the system, but its focus is more on guaranteeing high coverage and efficient bug finding rather than deriving quantitative measures (e.g., satisfaction probability) for a given property, as in SMC.

\section{MODELING FORMALISM}

We introduce our main modeling language, Probabilistic Timed I/O Automata with priorities and data (PTIOA), which extends Timed I/O Automata with priorities and data (TIOA) (Kwiatkowska et al. 2015; Barbot et al. 2016) with probabilistic delays.

TIOAs are well suited for modeling networked systems with real-time constraints and discrete control actions, as well as hybrid dynamics through continuous variables and nonlinear update functions, and thus provide an adequate level of representation for cardiac pacemaker models in a closed loop with hybrid models of the human heart (Kwiatkowska et al. 2015, Barbot et al. 2016). Importantly, this kind of automata can be expressed as MATLAB Stateflow diagrams (as shown by Barbot et al. 2015a, 2016), thus enabling effective tool support.

In addition, probabilistic features are essential to achieve personalization of heart models from data, as we will see in Section 4.2. By supporting arbitrary distributions to specify time delays, PTIOAs allow describing patients' features accounting for statistical information from the data.

We now provide a formal account of PTIOA and its semantics. In the following, we denote with $\operatorname{dist}(A)$ the set of probability distributions whose support is $A$.

Variables. A PTIOA includes a set of variables $V=\mathcal{X} \cup \mathcal{D}$, where $\mathcal{X}$ and $\mathcal{D}$ are the set of clocks and data, respectively. Clocks record the passage of time, while data variables can be updated to arbitrary real values. A variable valuation $\eta: V \rightarrow \mathbb{R}$ is a function that maps data variables to the reals and clocks to the nonnegative reals. For a set $\mathcal{Y}$, we denote with $\mathcal{V}(\mathcal{Y})$ the set of all valuations over $\mathcal{Y}$. For $\eta \in \mathcal{V}(V), \eta^{\mathcal{X}} \in \mathcal{V}(\mathcal{X})$ and $\eta^{\mathcal{D}} \in \mathcal{V}(\mathcal{D})$ denote the valuation $\eta$ restricted to clocks and data variables, respectively. The valuation $\eta$ after time $t \in \mathbb{R}_{\geq 0}$ has elapsed is denoted with $\eta+t$ and is such that $(\eta+t)(v)=\eta(v)+t$ if $v \in \mathcal{X}$ and $(\eta+t)(v)=\eta(v)$ otherwise. This captures the fact that all clocks proceed at the same speed and data variables are not affected by the passage of time.

Updates. In a PTIOA, variable valuations are manipulated through update functions. The update of a set of variables $V^{\prime} \subseteq V$ is a real-valued function $r: V^{\prime} \times \mathcal{V}(V) \rightarrow \mathbb{R}$. A valuation $\eta \in \mathcal{V}(V)$ is changed by the update function $r$ into the valuation $\eta[r]=\left\{v \mapsto r(v, \eta) \mid v \in V^{\prime}\right\} \cup\{v \mapsto \eta(v) \mid v \notin$ $\left.V^{\prime}\right\}$ that applies the update $r$ to the variables in $V^{\prime}$ and leaves the others unchanged. We denote with $\mathcal{R}$ the set of update functions.

Guards. We denote with $\mathcal{B}(V)$ the set of guard constraints over $V$, which describe the probabilistic firing conditions of automata edges. Specifically, guard constraints specify probabilistic delays of the form $g=\bigwedge_{i} x_{i} \geq t_{i}$, where $x_{i} \in \mathcal{X}$ is a clock and $t_{i} \sim D_{i}$ is a nonnegative random variable $^{2}$ describing the delay, and distributed according to the probability density function (PDF) $D_{i}: \mathcal{V}(\mathcal{D}) \rightarrow \operatorname{dist}\left(\mathbb{R}_{\geq 0}\right)$, which possibly depends on data variable valuations. Where possible, we alternatively use the shortcut $g=\bigwedge_{i} x_{i} \geq D_{i}$ in place of $g=\bigwedge_{i} x_{i} \geq t_{i}$, where $t_{i} \sim D_{i}$. Let $D_{i}\left(\eta^{\mathcal{D}}\right)$ be the PDF under valuation $\eta^{\mathcal{D}}$. We denote with $D_{i}^{\leq}\left(\eta^{\mathcal{D}}\right)$ the corresponding cumulative distribution function, i.e., such that for all $t \in \mathbb{R}_{\geq 0}, D_{i}^{\leq}\left(\eta^{\mathcal{D}}\right)(t)=\int_{-\infty}^{t} D_{i}\left(\eta^{\mathcal{D}}\right)(u) d u$.

${ }^{2}$ Not to be confused with the data variables. 
Remark 2.1 (Independence). We assume that the probabilistic delays in the guards of a PTIOA are mutually independent.

Under the above assumption, the satisfaction probability of guard $g$ under valuation $\eta \in \mathcal{V}(V)$ can be computed by factorizing the joint probability as follows:

$$
\operatorname{Pr}(\eta \mid=g)=\prod_{i} D_{i}^{\leq}\left(\eta^{\mathcal{D}}\right)\left(\eta\left(x_{i}\right)\right) .
$$

The random waiting time, $t(g, \eta)$, for $g$ to be satisfied under $\eta \in \mathcal{V}(V)$ is given by

$$
t(g, \eta)=\max \left(0, \max \left\{t_{i}-\eta\left(x_{i}\right)\right\}_{i}\right)
$$

Deterministic delays can be expressed by choosing a Dirac distribution for $D_{i}\left(\eta^{\mathcal{D}}\right)$. Note that, since $D_{i}$ can depend only on data variables, it is not affected by the passage of time, i.e., $\forall t \geq$ $0 . \forall \eta \in \mathcal{V}(V) . D_{i}\left((\eta+t)^{\mathcal{D}}\right)=D_{i}\left(\eta^{\mathcal{D}}\right)$.

Example 2.2 (Satisfaction Probability). Let $g=x \geq \mathcal{U}(1,2) \wedge y \geq \mathcal{U}(0,2)$, where $\mathcal{U}(a, b)$ is the uniform distribution on $[a, b]$, characterized by the cumulative distribution function $\mathcal{U} \leq(a, b)(x)=$ $\left\{0\right.$ if $x<a, \frac{x-a}{b-a}$ if $x \in[a, b], 1$ if $\left.x>b\right\}$.

Following Equation (1), the probability that valuation $\eta=\{x \mapsto 0, y \mapsto 0\}$ satisfies $g$ is 0 , since $\mathcal{U}^{\leq}(1,2)(0)=0$ and $\mathcal{U}^{\leq}(0,2)(0)=0$. The same holds for valuation $\eta^{\prime}=\{x \mapsto 1, y \mapsto 1.5\}$, since $\mathcal{U} \leq(1,2)(1)=0$. If from $\eta^{\prime}$ we let 0.5 time units pass, the corresponding valuation $\eta^{\prime}+0.5=\{x \mapsto$ $1.5, y \mapsto 2\}$ yields a satisfaction probability $\mathcal{U} \leq(1,2)(1.5) \cdot \mathcal{U} \leq(1,2)(2)=\frac{1}{2} \cdot 1$.

Remark 2.3 (Discrete Distributions). To simplify the presentation, probabilistic delays are defined over continuous distributions, even if the above definitions can be easily adapted to support discrete distributions.

Actions. Let $A$ be a set of action symbols. We consider the set of actions $\Sigma=\Sigma_{\text {in }} \cup \Sigma_{\text {out }}$, composed of input actions $\Sigma_{\text {in }}=\{? \alpha \mid \alpha \in A\}$ and output actions $\Sigma_{\text {out }}=\{! \alpha \mid \alpha \in A\}$. Each edge has an action associated, describing the event performed by that edge. As we shall see, automata components communicate by synchronizing on matching actions. For $a \in \Sigma_{\text {in }}$ and $b \in \Sigma_{\text {out }}$, we say that $a$ matches $b$ (or vice versa) if $a=? \alpha$ and $b=! \alpha$ for some $\alpha \in A$.

Definition 2.4 (PTIOA). A PTIOA with priority and data $\mathcal{A}=\left(X, \mathcal{D}, Q, q_{0}, \Sigma, \rightarrow\right)$ consists of

- a finite set of clocks $\mathcal{X}$ and data variables $\mathcal{D}$;

- a finite set of locations $Q$, with initial location $q_{0} \in Q$;

- a finite sets of input and output actions $\Sigma=\Sigma_{\text {in }} \cup \Sigma_{\text {out }}$; and

- a finite set of edges $\rightarrow \subseteq Q \times \Sigma \times \mathbb{N} \times \mathcal{B}(V) \times \mathcal{R} \times Q$. Each edge $e=\left(q, a, p r, g, r, q^{\prime}\right)$ is described by a source location $q$, an action a, a priority $p r$, a guard $g$, an update $r$, and a target $q^{\prime}$. Priorities define a total ordering of the edges out of any location, and are such that lower $p r$ values imply higher priorities.

Note that, unlike classical timed automata (Alur 1999), PTIOA locations do not include invariants in order to avoid time nondeterminism: as explained in Section 2.2, an enabled edge is fired as soon as possible, in a manner compatible with priority ordering, and no additional time can pass.

In addition to probabilistic dynamics, we remark that PTIOAs can express hybrid dynamics, and specifically hybrid automata whose differential equations admit explicit solutions that can be effectively computed. Indeed, we can use nonlinear update functions to encode such explicit solutions. 


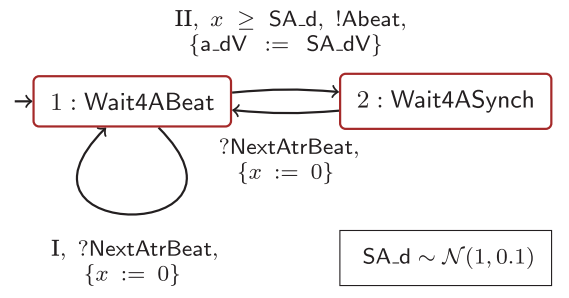

(a) SA node component

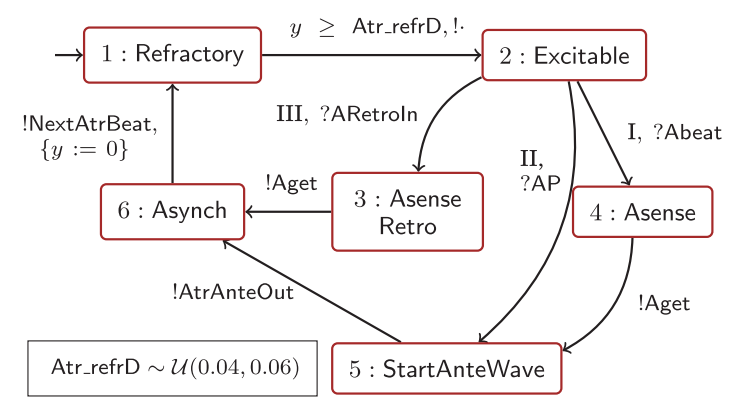

(b) Atrium component

Fig. 1. Portion of the PTIOA network of the heart model. Edge priorities are indicated with Roman numbers (I, II, III). Empty updates, trivially true guards, and priorities of edges with no alternative choices are omitted for clarity. Output action ! is used when no synchronization is sought for an edge.

\subsection{Networks of PTIOAs}

To facilitate modular designs, PTIOAs are able to synchronize on matching input and output actions, thus forming networks of communicating automata.

Definition 2.5 (Network of PTIOAs). A network of PTIOAs with $m$ components is a tuple $\mathcal{N}=$ $\left(\left\{\mathcal{A}^{1}, \ldots, \mathcal{A}^{m}\right\}, \mathcal{X}, \mathcal{D}, \Sigma\right)$, where

- for $j=1, \ldots, m, \mathcal{A}^{j}=\left(\mathcal{X}, \mathcal{D}, Q^{j}, q_{0}^{j}, \Sigma, \rightarrow^{j}\right)$ is a PTIOA, and

$-\mathcal{X}, \mathcal{D}$, and $\Sigma$ are the common sets of clocks, data variables, and actions, respectively.

We define the set of network modes by $\vec{Q}=Q^{1} \times \cdots \times Q^{m}$, with initial mode $\vec{q}_{0}=\left(q_{0}^{1}, \ldots, q_{0}^{m}\right)$ and the initial variable valuation $\eta_{0}$. For mode $\vec{q}=\left(q^{1}, \ldots, q^{m}\right) \in \vec{Q}$ and $j \in\{1, \ldots, m\}$, we denote with $\vec{q}_{j}=q^{j}$ the $j$ th component of $\vec{q}$, that is, the location of the $j$ th automaton. A state of the network is a pair $(\vec{q}, \eta)$, where $\vec{q} \in \vec{Q}$ is the vector of active locations and $\eta \in \mathcal{V}(V)$ is the variable valuation.

We use the notation $\mathcal{N}=\left(\left\{\mathcal{A}^{1}, \ldots, \mathcal{A}^{m}\right\}, \mathcal{X}, \mathcal{D}, \Sigma\right)$ to stress the fact that $\mathcal{X}, \mathcal{D}$, and $\Sigma$ are shared across $\mathcal{A}^{1}, \ldots, \mathcal{A}^{m}$.

Example 2.6 (PTIOA Network for SA Node and Atrium). Figure 1 shows a subnetwork of PTIOAs of the heart model, illustrated in Section 4.2. The automaton of Figure 1(a) describes the sinoatrial (SA) node, the component of the right atrium that generates intrinsic electrical stimuli. Figure 1(b) depicts the PTIOA for the atrium.

In the SA node component, after the firing period of the SA node has elapsed, modeled by the normally distributed delay SA_d $\sim \mathcal{N}(1,0.1)$ and guard $x \geq$ SA_d, the SA node transitions from the initial location Wait4ABeat to Wait4ASynch, performing an output action !Abeat to notify the other components of the network that the stimulus occurred. Data variable a_dV maintains the action potential of the signal and is updated to SA_dV, which is specific to stimuli generated from the SA node. In Wait4ASynch, the automaton waits until it can synchronize on action NextAtrBeat, at which point it resets the clock $x$ and returns to location Wait4ABeat.

In the atrium component, after a refractory period where the atrium cannot be stimulated, governed by the uniformly distributed delay Atr_refrD $\sim \mathcal{U}(0.04,0.06)$, the atrium becomes excitable and can receive three types of impulses: an intrinsic stimulus from the SA node, through synchronization on Abeat; a paced stimulus from the pacemaker (AP); or a retrograde signal from the ventricles (AtrRetroln). These trigger a contraction of the atria. When the atrial stimulus is generated by the SA node or the pacemaker, this is propagated toward the ventricles through the 
AV node by performing action !AtrAnteOut. When the stimulus is not artificial, the component emits an Aget action for the pacemaker to read. Before reaching the Refractory location again, an intermediate action is required (!NextAtrBeat) to synchronize the SA node component.

Note that, while the VVIR pacemaker under study stimulates only the ventricle, the Atrium component supports atrial pacing, and thus can be also connected to models of atrial or dualchamber pacemakers.

In this example, we arbitrarily chose the random delay SA_d to produce a heart period with mean 1s (i.e., rate of $60 \mathrm{BPM}$ ). In our experiments, however, SA_d depends on the ideal rate demand, and thus on the kind of activity performed, as explained in Section 4.2.3. On the other hand, the atrial refractory period Atr_refrD is based on the original parameterization of Barbot et al. (2015b).

\subsection{Semantics}

Enabled Components and Synchronization. Let $\mathcal{N}=\left(\left\{\mathcal{A}^{1}, \ldots, \mathcal{A}^{m}\right\}, \mathcal{X}, \mathcal{D}, \Sigma\right)$ be a PTIOA network and $(\vec{q}, \eta)$ be its current state. We say that a PTIOA component $\mathcal{A}^{j}$ of $\mathcal{N}$ is enabled from $(\vec{q}, \eta)$ if, from its current location $\vec{q}_{j}$, it has at least one enabled edge. An output edge is enabled when the associated guard is satisfied under valuation $\eta^{3}$, while an input edge is enabled when its guard holds under $\eta$ and, at the same time, it can synchronize with a matching output action fired by another component of the network. This means that, unlike output edges, input edges can fire only by synchronizing with a matching output action; i.e., an edge labeled with $! \alpha$ can fire without other components firing ? $\alpha$, but not vice versa. In turn, we allow for multiparty synchronization; that is, multiple input edges can synchronize with a single matching output edge.

Product PTIOA. Formally, the semantics of a PTIOA network is defined over the corresponding product PTIOA (see Supplementary Material, Section 1 for details on its construction). The product PTIOA of a network $\mathcal{N}$ is a PTIOA $\mathcal{N}_{\otimes}=\left(X, \mathcal{D}, \vec{Q}, \vec{q}_{0}, \mathcal{P}\left(\Sigma_{\text {out }}\right), \rightarrow\right)$, where locations correspond to the set of network modes $\vec{Q}$, and edges in $\rightarrow$ are defined by applying the above synchronization rules. To reflect the simultaneous firing of multiple components, an edge $e=(\vec{s}, a, p r, g, r, \vec{t}) \in \rightarrow$ is characterized by an action $a \in \mathcal{P}\left(\Sigma_{\text {out }}\right)$ given by the set of output actions fired by the enabled components; a vector of priorities $p r=\left(p r^{1}, \ldots, p r^{m}\right) \in \mathbb{N}^{m}$, where, for $j=1, \ldots, m, p r^{j}$ is the priority of the edge fired by $\mathcal{A}^{j}$ or $+\infty$ if $\mathcal{A}^{j}$ is not enabled; and a guard $g$ and an update $r$ obtained by combining guards and updates, respectively, of the fired edges.

Below, we describe the semantics of PTIOA networks in a way that facilitates their encoding into Discrete Event Stochastic Processes (Ballarini et al. 2015), for which we can leverage efficient statistical model checking algorithms, as described in Section 3.

Semantics. Given a PTIOA network $\mathcal{N}$ and its product PTIOA $\mathcal{N}_{\otimes}=\left(\mathcal{X}, \mathcal{D}, \vec{Q}, \vec{q}_{0}, \mathcal{P}\left(\Sigma_{\text {out }}\right), \rightarrow\right)$, the semantics of $\mathcal{N}$ is described by timed paths of the form $\rho=\left(\vec{q}_{0}, \eta_{0}\right) \stackrel{e_{0}, t_{0}}{\longrightarrow}\left(\vec{q}_{1}, \eta_{1}\right) \stackrel{e_{1}, t_{1}}{\longrightarrow} \cdots$, where, for each $i, \rho[i]=\left(\vec{q}_{i}, \eta_{i}\right)$ is a state of the network, $t_{i}$ is the time spent in that state, and $e_{i} \in \rightarrow$ is the edge fired in $\mathcal{N}_{\otimes}$.

Given the probabilistic nature of the model, $\rho[i]=\left(\vec{q}_{i}, \eta_{i}\right), t_{i}$ and $e_{i}$ are families of random variables, characterized for each step $i$ as follows (see Supplementary Material, Section 1 for a formal definition). Let $t\left(e, \eta_{i}\right)$ be a random variable describing the waiting time for $e \in \rightarrow$ to be enabled under valuation $\eta_{i}$ (see Equation (2)). Then, the waiting time $t_{i}$ is the shortest waiting time among the outgoing edges of $\vec{q}_{i}$, and $e_{i} \in \rightarrow$ is the edge with the shortest waiting time and highest priority. Finally, the next state $\rho[i+1]=\left(\vec{q}_{i+1}, \eta_{i+1}\right)$ is determined by the target location and the update of $e_{i}$.

\footnotetext{
${ }^{3}$ To simplify the presentation, here guard satisfaction is assumed under some realization (sampling) of the random delays associated to the guard constraint.
} 


\section{STATISTICAL MODEL CHECKING WITH HASL PROPERTIES}

For the verification of PTIOA models we employ SMC, a technique for the verification of probabilistic properties based on simulating the system for finitely many runs (Younes et al. 2006). This provides an effective, yet approximate, verification method for PTIOA networks, for which no precise (numerical) model checking method exists. As we shall see, another advantage of SMC is that it supports rich specification languages, including quantitative and automata-based properties.

In this work, we consider properties specified in the Hybrid Automata Stochastic Language (HASL) (Ballarini et al. 2015), implemented in the statistical model checker Cosmos. ${ }^{4}$ An HASL formula consists of two parts:

(1) A Linear Hybrid Automaton (LHA) that keeps a set of data variables and synchronizes with executions of the system, in our case paths of a PTIOA network.

(2) The actual quantity to evaluate, given as an expression over the data variables of the LHA.

In the following, we give a brief overview of these two components. For a detailed explanation, we refer to Ballarini et al. (2015) and Supplementary Material, Section 2.

Linear Hybrid Automata. We outline the main differences between LHAs and PTIOAs (introduced in the previous section). Since an LHA is meant to synchronize with the paths of the PTIOA network under analysis, it also contains a final location that determines when to terminate and accept the current path. If at any point the LHA cannot synchronize with the PTIOA network, the path is terminated and rejected. In particular, an LHA edge can either synchronize with a set of PTIOA actions ${ }^{5}$ or proceed autonomously-i.e., it can fire without synchronization (denoted by action \#). Unlike PTIOAs, LHA guards are deterministic and are specified as linear constraints over LHA variables. In addition to update functions, the evolution of LHA variables is controlled by flows, i.e., linear functions describing their change rate at each location.

HASL Expressions. We introduce the HASL fragment of interest for our properties (see Appen$\operatorname{dix} \mathrm{B}$ for the full syntax). A HASL expression is of the form $\mathrm{E}[Y]$ and allows for evaluating the expectation of a so-called path variable $Y=\operatorname{LAST}(y)$, i.e., a random variable denoting the value of $y$ at the final state of the synchronized execution.

The term $y$ is an arithmetic expression over LHA variables, built using the operations $\{+, \cdot,-, /\}$. A derived HASL expression is $\operatorname{PDF}(Y, h, a, b)$ that computes a discrete approximation of the probability density function (PDF) of path variable $Y$ in the interval $[a, b]$ and using $h$ subdomains.

In the Cosmos tool, statistical model checking of a HASL expression $Z$ is based on confidence interval estimation: given a confidence level $\alpha \in(0,1)$ and number of executions $n$, the method returns a confidence interval $\mathrm{CI}_{\mu_{Z}}$ for the expected value of $Z, \mu_{Z}$, where $\alpha$ represents the frequency of possible confidence intervals that contain the actual value of $\mu_{Z}$. The method also returns an estimator for $\mu_{Z}$, given by the mean of the values of $Z$ over the above $n$ executions.

Example 3.1 (Fraction of Paced Beats). Consider the LHA in Figure 2. Its purpose is to record the number of paced and total ventricular beats occurring within Tmax time units. These quantities are kept respectively in data variables VPs and Nbeats. Clock $t$ stores the current time. All variables are initialized to 0 . Flows indicate that VPs and Nbeats have associated a change rate of 0 , while $t$ has a change rate of 1 . The LHA is intended to synchronize with the heart and pacemaker network presented in Section 4.2, where intrinsic and paced ventricular impulses are implemented through actions Vget and VP, respectively.

\footnotetext{
${ }^{4}$ Available at http://www.lsv.ens-cachan.fr/Software/cosmos/.

${ }^{5}$ Since they synchronize with the output actions fired by the PTIOA network, all LHA actions are implicitly input actions.
} 


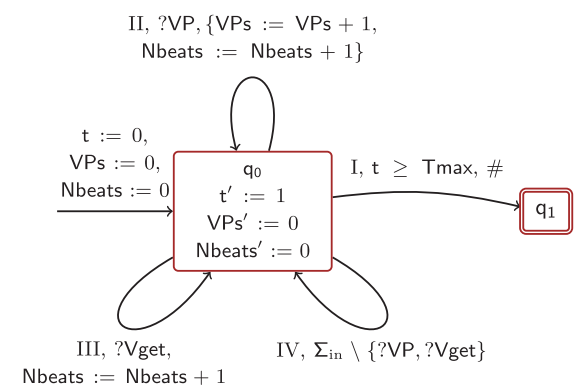

Fig. 2. LHA for estimating the fraction of paced beats. Final locations are denoted with double-bordered boxes.

The automaton has two locations: an initial location $q_{0}$ and a final location $q_{1}$, reached from $q_{0}$ with an autonomous edge as soon as $t \geq$ Tmax.

Consider the following path, describing the antegrade propagation of the action potential from the atrium to the ventricle, as shown in the high-level schema of the heart model of Figure $4:^{6}$

$$
\begin{aligned}
& \left(\vec{q}_{0}, \eta_{0}\right) \stackrel{\text { :Abeat, } 0.9}{\longrightarrow}\left(\vec{q}_{1}, \eta_{1}\right) \stackrel{! \text { Aget, } 0}{\longrightarrow}\left(\vec{q}_{2}, \eta_{2}\right) \stackrel{\text { :AtrAnteOut, } 0}{\longrightarrow}\left(\vec{q}_{3}, \eta_{3}\right) \stackrel{\text { !NextAtrBeat, } 0}{\longrightarrow}\left(\vec{q}_{4}, \eta_{4}\right) \stackrel{\text { !AVJAnteln, } 0.03}{\longrightarrow} \ldots \\
& \ldots\left(\vec{q}_{5}, \eta_{5}\right) \stackrel{\text { !AVJAnteOut, } 0.02}{\longrightarrow}\left(\vec{q}_{6}, \eta_{6}\right) \stackrel{\text { !AVVAnteln, } 0.04}{\longrightarrow}\left(\vec{q}_{7}, \eta_{7}\right) \stackrel{\text { !VtrAnteln, } 0.05}{\longrightarrow}\left(\vec{q}_{8}, \eta_{8}\right) \stackrel{\text { !Vget, } 0}{\longrightarrow}\left(\vec{q}_{9}, \eta_{9}\right) .
\end{aligned}
$$

Let Tmax $=1.5$. From the initial location $q_{0}$, the LHA synchronizes with path transitions $\stackrel{e_{0}, t_{0}}{\longrightarrow}$ $\ldots \stackrel{e_{7}, t_{7}}{\longrightarrow}$ by firing the loop labeled with $\Sigma_{\text {in }} \backslash\{$ ?VP, ?Vget $\}$. This edge has the least priority, a trivially true-guard, empty update, and can match any output action fired by the PTIOA network apart from ?VP and ?Vget. Without such an edge, the LHA would fail to synchronize at the first transition (labeled with \{!Abeat\}), and the path would be immediately rejected. Along transition $\stackrel{e_{8}, t_{8}}{\longrightarrow}$, the PTIOA network fires $\{!$ Vget $\}$, leading to a synchronization on the LHA edge labeled with ?Vget. The resulting variable valuation is $t=1.04$, Nbeats $=1$, and VPs $=0$.

Consider the continuation $\rho=\ldots\left(\vec{q}_{9}, \eta_{9}\right) \stackrel{\text { IVP, } 0.3}{\longrightarrow}\left(\vec{q}_{10}, \eta_{10}\right)$. Here, the LHA synchronizes with $\stackrel{e_{9}, t_{9}}{\longrightarrow}$ on the LHA edge labeled with ?VP, leading to valuation $t=1.34$, Nbeats $=2$, and VPs $=1$. After time Tmax $-t=0.16$, the LHA can fire the autonomous edge to the final location. Assuming no !VP or !Vget is emitted by the PTIOA network during this period, the final valuation is $t=1.5$, Nbeats $=2$, and $\mathrm{VPs}=1$.

Let $y=\frac{\mathrm{VPs}}{\text { Nbeats }}$ denote the fraction of paced beats over the total number of beats. For path $\rho$, $\operatorname{LAST}(y)=\frac{1}{2}$, because Nbeats $=2$ and VPs $=1$ at the final state. A property that we will analyze in Section 5 is the PDF of the fraction of paced beats at the end of the execution, i.e., after time Tmax:

$$
\phi_{\mathrm{PDF}(\mathrm{VP})}=\operatorname{PDF}\left(\operatorname{LAST}\left(\frac{\mathrm{VPs}}{\text { Nbeats }}\right), h, a, b\right),
$$

where $a=0$ and $b=1$, given that the ratio $\frac{\mathrm{VPs}}{\text { Nbeats }}$ ranges in the interval $[0,1]$. We further set $h=0.01$, which corresponds to dividing the domain of the PDF in 100 subdomains. ${ }^{6}$ With abuse of notation, we denote transition $\stackrel{e, t}{\longrightarrow}$ by $\stackrel{a(e), t}{\longrightarrow}$, where we replace the edge $e$ with the corresponding set of
fired actions $a(e)$. 


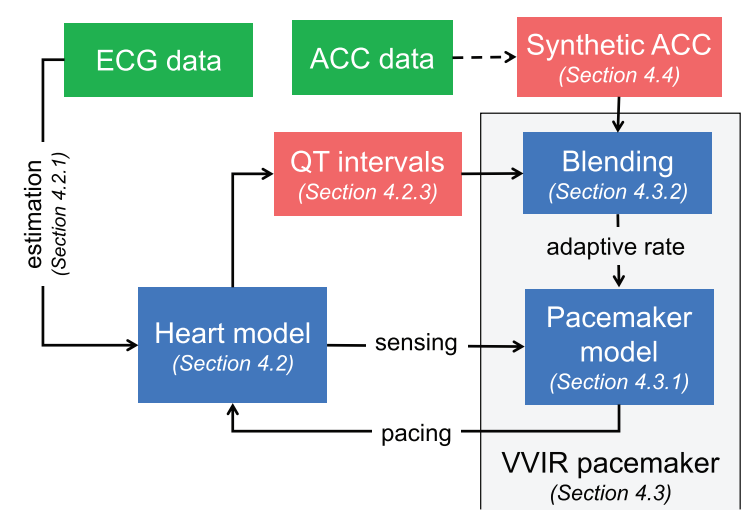

Fig. 3. Closed-loop architecture for rate adaptation.

\section{MODELS AND METHODS}

In this section, we introduce our closed-loop design for the rate-adaptive pacemaker and describe the heart and pacemaker models, the algorithms for the processing of physiological data, and how the extracted features are combined to compute an adequate adaptive rate. The design, illustrated in Figure 3, realizes a dual-sensor VVIR pacemaker, that is, a pacemaker that senses and paces only the ventricle and supports rate adaptation. The heart model (explained in Section 4.2) and the pacemaker model (Section 4.3) are connected into a closed-loop system and implemented as a PTIOA network, where these two components communicate with each other to detect intrinsic heartbeats (sensing) and deliver artificial beats (pacing). We include a so-called sensor blending algorithm (Section 4.3.1) that computes the adaptive pacing rate by combining QT-based and accelerometer sensors. The QT sensor exploits the fact that physical and mental stress shortens the QTI. The blending algorithm estimates patient-specific mathematical laws between the QTI and the heart period (also called RR interval) in order to predict an adequate pacing rate based on the QTI measured by the pacemaker electrogram.

To close the loop between the heart dynamics and the sensor data used by the blending algorithm, we generate online, synthetic QTIs (Section 4.2.3) that reflect the state of the heart model simulation. We further devise a method for generating synthetic accelerometer signals, whose parameters can be either estimated from offline recordings in order to reproduce patient-specific characteristics or configured to reproduce specific physical activities, e.g., running or walking.

Through the parameter estimation method of Section 4.2.1, our approach crucially enables the derivation of personalized heart models from patient ECG data, resulting in patient-specific virtual patient models. Importantly, if we use ECG data from a set of multiple patients instead of a single patient, we can apply the same method to estimate virtual populations of patients, i.e., models able to fully capture the varied characteristics of the input population. Therefore, the synthetic QTIs received by the blending algorithm in turn reflect the features of the input patient/population, since they are generated from the corresponding virtual patient/population.

We remark that parameter estimation is performed offline, prior to the closed-loop analysis, and that this results in a probabilistic heart model, which motivates the application of quantitative verification techniques.

Open-Loop Variant. To assess the effectiveness of our closed-loop design, we also consider an open-loop variant, where the QTIs are not generated from the model but are extracted from the input (offline) ECG data. Such obtained QTIs are fixed, implying that the adaptive rate does not 
Table 1. Age (y) and HR at Rest

(HR $\mathrm{HR}_{\text {rest }}$, BPM) for the Virtual

Patients of Our Study

\begin{tabular}{r|cc}
\hline & Age & $\mathrm{HR}_{\text {rest }}$ \\
\hline Subject 1 & 21 & 80 \\
Subject 2 & $34^{(*)}$ & 75 \\
Subject 3 & 52 & 67 \\
V. population & $34^{(*)}$ & 77 \\
\hline
\end{tabular}

${ }^{(*)}$ Due to the anonymity of the record, the age is set to the median of the ages of the participants in Anguita et al. (2013).

reflect the dynamic state of the heart model. For this reason, compared to the open-loop variant, the closed-loop design can reproduce with superior accuracy phenomena of sensor-induced endlessloop tachycardia, as we will see in the experimental evaluation section.

We first introduce the virtual patients over which we evaluate our design, and the corresponding input data for parameter estimation.

\subsection{Virtual Patients}

We consider two kinds of heart models: patient-specific models for the analysis of personalized treatments (Subjects 1-3 below) and population-level models, which can capture the varied features of a pool of patients, and thus are suitable for safety verification (Virtual population below).

Subject 1. The data consists of one-lead ECG and one-axis accelerometer data obtained from a volunteer (one of the authors) and recorded through a BITalino board. ${ }^{7}$ We performed recordings at rest and at varying levels of activity, for a total of 15 minutes.

Subject 2. The data corresponds to record 16272 of the MIT-BIH Normal Sinus Rhythm Database (Goldberger et al. 2000), consisting of 1-hour, two-lead ECG recordings of patients without any particular arrhythmia. Since we do not have accelerometer data for this subject, we generate it following the synthetic generation method discussed in Section 4.4, and using data from Anguita et al. (2013).

Subject 3. Signals are extracted from the data published by Alan Dix, ${ }^{8}$ collected during his $1,000+$ miles circumlocution of Wales. Public data consists of 35 sequences of 40 hours of ECG (one-lead) and three-axis accelerometer signals, recorded through wearable devices during resting, activity, and sleep. Of this database, we consider an extract of day 1.

Virtual Population. We estimate a virtual population of patients based on 10 subjects taken from the MIT-BIH ST Change Database (Albrecht 1983), ${ }^{9}$ which consists of ECG recordings of different patients during exercise stress tests. Similarly to Subject 2, we generate accelerometer signals based on the data from Anguita et al. (2013). In Table 1, we also report age and HR at rest of the above four patients. As we will see in Section 4.3.3, these parameters are used within the rate adaptation algorithm.

\footnotetext{
${ }^{7}$ http://www.bitalino.com/

${ }^{8}$ http://alanwalks.wales/data/

${ }^{9}$ IDs: 300, 301, 302, 304, 307, 308, 310, 311, 312, 313.
} 


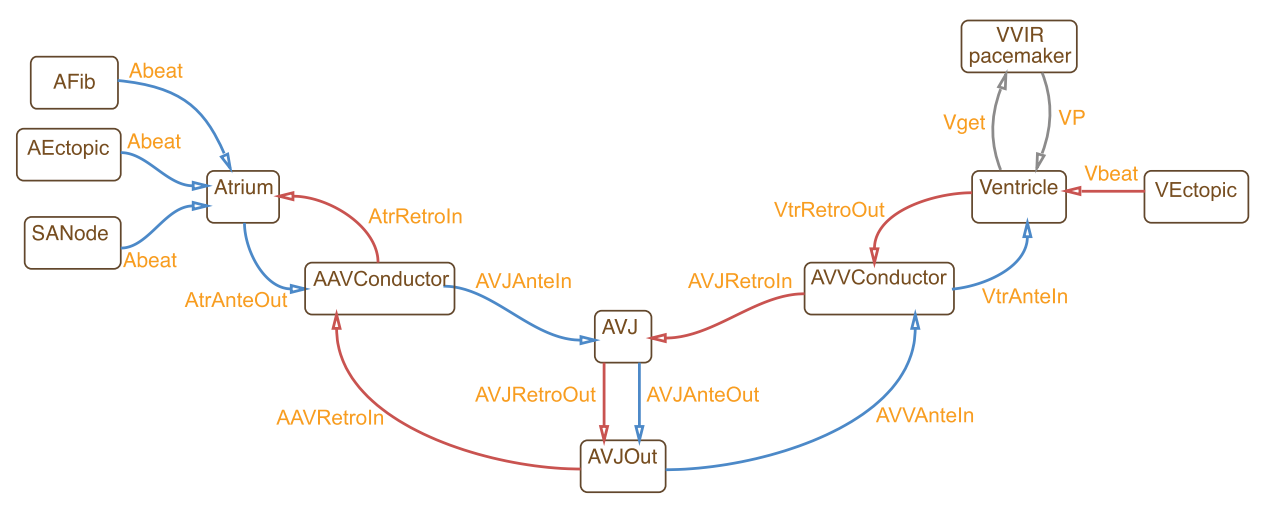

Fig. 4. High-level view of the PTIOA components of the heart model. Arrows indicate synchronizations, with the target component synchronizing with the output action performed by the source component, and are labeled with the action name. The antegrade conduction (blue arrows) passes from the Atrium to the Ventricle component. The retrograde conduction (red arrows) follows the opposite path. Gray arrows indicate the connection with the VVIR pacemaker.

We remark that ECG recordings are only used to estimate the parameters of the heart model, while the closed-loop simulation just relies on synthetic data (QTIs and accelerometer).

\subsection{Heart Model}

Below, we illustrate the heart model, including the method for estimation from ECG data, the modeling of relevant arrhythmias, and the online generation of synthetic QTIs. The model reproduces the electrical conduction system of the heart, that is, the propagation of the cardiac action potential (AP) from the atria (the upper chambers) to the ventricles (the lower chambers) through the atrioventricular (AV) node. Here, we only provide a high-level view of the model, which is depicted in Figure 4. For a comprehensive description of the model components, we refer to the original publication (Barbot et al. 2015a) and Supplementary Material, Section 6.

The model consists of 10 conduction nodes and two main conduction pathways: the antegrade conduction, that is, the normal situation where an electrical impulse generated by the SA node stimulates the atria and is conducted to the ventricles through the AV node (component AVJ in Figure 4), and the retrograde conduction, where the impulse travels in the opposite direction (from the ventricles to the atria through the AV node). Generally, retrograde conduction is less frequent and originates when the ventricular myocardium is stimulated artificially by a pacemaker or by an ectopic action potential. The conduction between nodes is implemented through synchronization between the involved components. In this way, the model can be easily extended with other accessory conduction pathways.

In the figure, we also illustrate the connection with the pacemaker component: the device sends impulses to the right ventricle through action VP and senses intrinsic impulses from the right ventricle by synchronizing on action Vget.

We have already inspected the Atrium and SANode PTIOAs in Example 2.6.

Component AEctopic generates the so-called atrial ectopic beats, which originate from the spontaneous excitation of different portions of the atrial tissue, mainly from the pulmonary veins, but are not as frequent as the stimuli from the SA node. A dedicated component, AFib, is included to model atrial fibrillation, as we will see in Section 4.2.2.

The Ventricle and VEctopic components describe the AP dynamics in the ventricles, and are modeled in a similar way to the Atrium and AEctopic components, respectively. There are two 


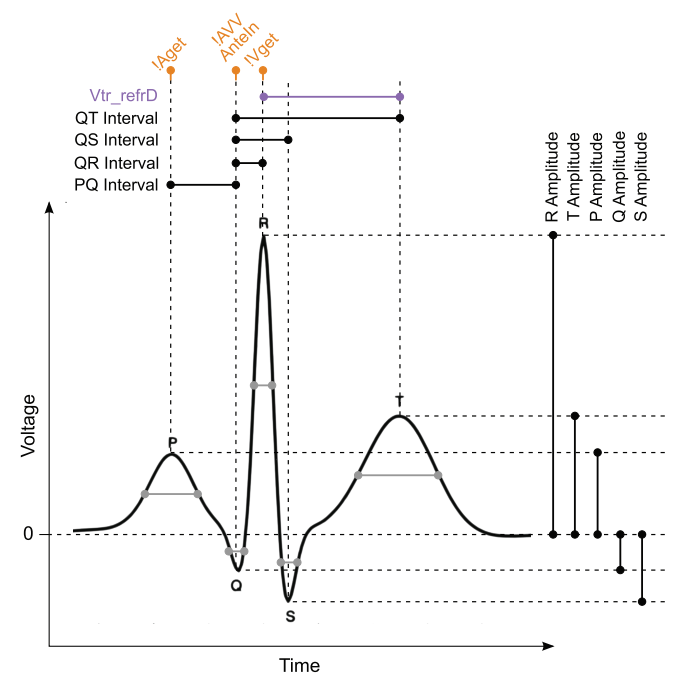

Fig. 5. Example of ECG for one cardiac cycle and corresponding features. Top: Duration features, and mappings with actions (orange) and delay parameters (purple) of the heart model. Right: Amplitude features. For each wave, the gray solid line indicates the width at half amplitude. Vtr_refrD is the ventricular refractory period.

structurally equivalent conductor components, AAVConductor (Atrium-AV node conductor) and AVVConductor (AV node-Ventricle conductor), whose role is to apply a propagation delay to the transmission of the action potential in both directions. From a physiological viewpoint, the AAVConductor represents the so-called internodal tracts, while the AVVConductor is an abstraction of the nodes connecting the AV node and the ventricles, namely, the bundle of His and the Purkinje fibers. The AV node is modeled through components AVJ and AVJOut. Unlike the other components that use delays to implement the conduction of cardiac waves, AVJ provides a detailed representation of the action potential dynamics, whose conformation is reproduced through nonlinear update functions. This enables the modeling of complex conditions such as the AV block, as explained below. Component AVJOut applies additional delays depending on the state of the AV node action potential.

Delays in the heart network are mainly probabilistic, as a result of the estimation from ECG data described below and in detail in Barbot et al. (2015a).

4.2.1 Personalization. The ECG is composed of five main waves, $\mathrm{P}, \mathrm{Q}, \mathrm{R}, \mathrm{S}$, and $\mathrm{T}$, which correspond to specific heart events, as illustrated in Figure 5 and explained in Malmivuo and Plonsey (1995), Chapter 15. In particular, time-domain ECG features can be mapped into specific actions and parameters in our heart model:

- The $\mathrm{P}$ wave corresponds to the activation and propagation of the AP in the atria. In the heart model, the event !Aget captures the moment at which an atrial pacemaker lead would sense the signal, which happens as the ECG signal rises toward the P peak. We assume that !Aget coincides with the $\mathrm{P}$ peak.

- The $Q$ wave corresponds to the AP propagating into the Purkinje fibers and the inner walls of the ventricles. In our model, this is equivalent to the event !AVVAnteln, which indicates the beginning of the AP propagation into the AVV conductor component. 
- The QRS complex corresponds to the propagation of the depolarization wave into the ventricles. Similarly to the $\mathrm{P}$ wave in the atria and action !Aget, we assume that action !Vget (denoting AP sensing by the ventricular lead) corresponds exactly to the R peak.

- Finally, the $\mathrm{T}$ wave is generated by the ventricle repolarization wave. The $\mathrm{T}$ peak corresponds to the end of the ventricular absolute refractory period, which is indicated by the delay parameter, Vtr_refrD, in our model.

Note that the PTIOA heart model does not distinguish between the left and right ventricle, meaning that it does not have actions or timing delays mapping into the $S$ wave of the ECG, which roughly corresponds to the depolarization of the left ventricle.

Importantly, through this mapping between the heart model and the ECG, the synthetic QTI (used as a sensor for rate adaptation) is derived as the time elapsed between the event !AVVAnteln and the ending of the corresponding ventricular refractory period. Details of the generation of synthetic QTIs are given in Section 4.2.3.

Our parameter estimation method relies on the generation of synthetic ECG signals (following the method of McSharry et al. (2003), Barbot et al. (2015b), and Eberz et al. (2017)) that resemble the behavior of the electrical propagation in the heart during the execution of the model. Such synthetic ECGs are synchronized with the heart model simulation according to the above-explained mapping, and are obtained by summing, for each wave kind, a Gaussian curve that reproduce the morphology of the wave.

In particular, estimation of patient-specific models from ECG data consists of the following two steps:

1) Extraction of ECG features. This step results in a set of discrete probability distributions $\left\{D_{f}\right\}_{f}$, one for each ECG feature $f$. The features considered are those of Figure 5. Following the mapping described above, some of the model parameters can be directly estimated at this stage, in terms of probabilistic delays distributed according to the relevant feature distribution $D_{f}$. For feature extraction, we combine the Pan-Tompkins peak detection algorithm (Pan and Tompkins 1985) to identify R peaks with local search to identify the other peaks.

2) Estimation of "hidden" parameters. We estimate the heart parameters that cannot be directly derived from any ECG feature, hence called hidden. This boils down to finding the hidden parameter values that minimize a suitable statistical distance between the input ECG and the synthetic ECG generated from the model simulation. In addition to the explicit parameters of step 1, we estimate two hidden parameters, the AV node threshold voltage AV_Vt and the minimum conduction time in the AV node AV_DMin, because these were the conduction parameters that produced the highest sensitivity for the synthetic ECG. We consider the statistical distance of Eberz et al. (2017), which is computed as the mean of the statistical distances between the feature distributions, and solve the optimization problem using the pattern search algorithm (Kolda et al. 2003), even though our method supports arbitrary (black-box) optimization algorithms and distances.

Estimation for Virtual Population Models. In order to estimate the parameters of virtual population models, we proceed by performing steps (1) and (2) for each patient of the input dataset. This results in a set of random variables $x_{i, p} \sim D_{i, p}$, describing the values of each estimated parameter $i$ for each patient $p$. Then, the virtual population model is obtained by combining, for each parameter, the patient-specific distributions across all patients into a single distribution: parameter $i$ is characterized by random variables $x_{i} \sim D_{i}$, where $D_{i}=\biguplus_{p} D_{i, p}$ is the combined distribution. ${ }^{10}$ Virtual population models can thus represent all the dynamics that could result from each of their

\footnotetext{
${ }^{10}$ For simplicity, the combined distribution is defined as the multiset sum of the underlying samples.
} 


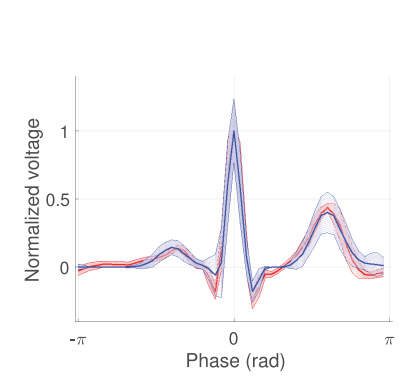

(a) Subject 1

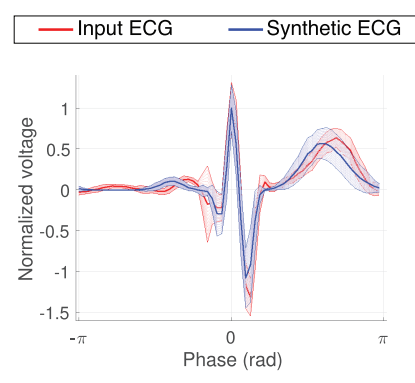

(b) Subject 2

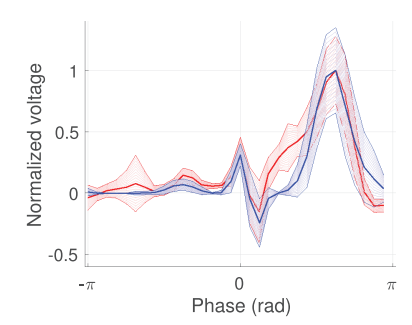

(c) Subject 3

Fig. 6. Comparison of mean input ECG and mean synthetic ECG for the estimated patient-specific models. Shaded areas span \pm 1 standard deviation around the mean. Mean ECGs are computed after a linear phase assignment (Sameni et al. 2007), assigning a periodic phase value to each sample in the ECG, starting from one R-peak (phase 0 ) and ending with the next R-peak (phase $2 \pi$ ). For each heart cycle, amplitudes are normalized by the amplitude of the corresponding highest peak.

constituent patients, as well as new dynamics that may arise from the "cross-talking" among the individually estimated patient-specific features.

Figure 6 compares the input ECG signals used for the estimation and the corresponding synthetic ECG produced by a simulation trace of the model, after the parameter estimation is performed. We observe good agreement between the two, especially as far as time-domain features (i.e., time between peaks) are concerned, which is most relevant for our PTIOA model. The main difference is in the shape of the $\mathrm{T}$ wave, which is naturally asymmetric, while our synthetic ECG waves are generated as symmetric Gaussian curves. We remark, however, that this lack of asymmetry does not affect the estimated time delays, and that it can be easily accounted for by introducing an additional width feature, as done in Eberz et al. (2017).

4.2.2 Heart Conditions. The VVIR pacing mode is generally recommended for patients suffering from both AV block and SA node dysfunctions, or chronic atrial impairment (e.g., atrial fibrillation or flutter) (Iaizzo 2009, Fig. 30.7-8). Therefore, in our model we reproduce AV block and atrial fibrillation $(\mathrm{AF})$ as illustrated below.

AV Block. we consider the heart disorder called Wenckebach AV block (Hampton and Adlam 2013), a conduction failure causing the loss of ventricular beats due to the progressive prolongation of the AV conduction time. The Wenckebach AV block is a form of chronotropic incompetence typically addressed by setting the pacemaker to the rate-adaptive mode. As illustrated in Barbot et al. (2015a), this condition can be reproduced in our heart model by increasing parameter AV_Vt, i.e., the depolarization threshold potential of the AV node. We stress that, although we arbitrarily introduce AV block, this does not compromise the original electrophysiological characteristics of the patient. Indeed, the Wenckebach AV block affects only the duration of the PQ interval (Hampton and Adlam 2013, Chapter 4), and thus has no effect on the other ECG features, including the QTI. In the heart model, the AV block prolongs the timing delays solely of the AVJ component, meaning that all the other components maintain the subject-specific behavior learned from ECG data.

Atrial Fibrillation. AF is the most common cardiac arrhythmia (Markides and Schilling 2003) and is characterized by a highly disorganized atrial electrical activity, which is thought to be caused by the interaction of rapidly firing ectopic extra-atrial foci and abnormal atrial tissue. We model AF through the AFib component (depicted in Figure 7), which is responsible for the generation of random AF impulses. Following Lian et al. (2006), the irregular rates of the electrical signals are 


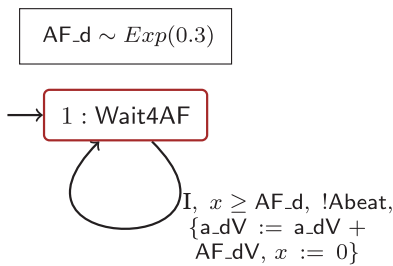

Fig. 7. AFib component.

governed by an exponential distribution (with mean $0.3 \mathrm{~s}$ ), and the corresponding action potential $\left(A F \_d V\right)$ is not strong enough to stimulate, by itself, the AV node.

For the purpose of model personalization from ECG data, we assume that AF is either recurrent (i.e., few AF episodes occurred to the patient), paroxysmal (i.e., repeated self-terminating AF episodes that last less than 7 days), or persistent (i.e., long-lasting AF episodes that can be terminated by external intervention) (Markides and Schilling 2003). Indeed, in all these cases we can obtain ECG segments showing no AF episodes, and use them to estimate the model parameters and the subject-specific relationship between QT and RR (see Sections 4.3.2 and 4.2.3). For permanent AF (i.e., long-lasting AF episodes that cannot be terminated by external intervention), we cannot obtain useful ECG segments to learn the relationship between QT and RR intervals, which is at the basis of rate adaptation. In this case, however, we can still use a different rate adaptation model that works for virtual populations and does not require patient-specific data, as explained in Section 4.3.2.

4.2.3 Synthetic QT Intervals. As previously explained, there is a clear patient-specific correlation between the QT and the RR intervals in every heartbeat, which is exploited by the blending algorithm to compute the adaptive rate (see Section 4.3.1). Therefore, it is crucial to account for the QT-RR relationship also in our heart model, so that the synthetic QTIs used for rate adaptation reflect this correlation in an accurate way and adapt to the dynamical HR changes due to, e.g., exercise, pacing, or conduction defects. To this purpose, we derive from the input ECG data a joint discrete probability distribution, denoted by $D_{\mathrm{QT}, \mathrm{RR}}: \mathbb{R}^{2} \rightarrow[0,1]$, which characterizes the observed QT and RR intervals at each heart cycle. For virtual population models, the distribution is obtained by combining the set of samples across the multiple-input datasets.

The actual dynamics of the QTI shortening depend on both the effective HR and the autonomic tone (Ahnve and Vallin 1982; Davey and Bateman 1999). The autonomic tone resembles the ideal rate demand, which is represented in our model by the firing period of the SA node SA_d (see the SA node model of Figure 1(a)). However, the electrical stimuli produced by the SA node are overwhelmed by the effect of $\mathrm{AF}$, and are also affected by the AV conduction defects, thus producing a discrepancy between the ideal rate demand and the effective HR in cardiac patients. On the basis of the experimental results obtained by Magnano et al. (2002), we assume that the QT interval correlates with both the ideal rate demand and effective HR.

Consider a generic heart cycle $t$, and let $\overline{\mathrm{HR}}_{t}$ be the corresponding ideal rate demand (in BPM), which depends on the current activity level. The synthetic QTI at $t, \mathrm{QT}_{t}$, is generated as follows:

(1) We update the SA node firing period based on the ideal rate: SA_d $t=60 / \overline{\mathrm{HR}}_{t}$.

(2) To consider the combined effect of the effective and ideal HR, we define $\overline{\mathrm{RR}}_{t}$ as the weighted sum $\overline{\mathrm{RR}}_{t}=k \cdot \mathrm{SA} \_\mathrm{d}_{t}+(1-k) \cdot \mathrm{RR}_{t-1}$, where $k \in(0,1)$ and $\mathrm{RR}_{t-1}$ is the effective heart period at $t-1$, i.e., computed as the time between the last two ventricular events.

(3) $\mathrm{QT}_{t}$ is finally sampled from distribution $D_{\mathrm{QT}, \mathrm{RR}}$, conditioned on $\mathrm{RR}=\overline{\mathrm{RR}}_{t}$. 


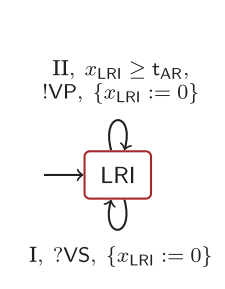

(a) LRI component

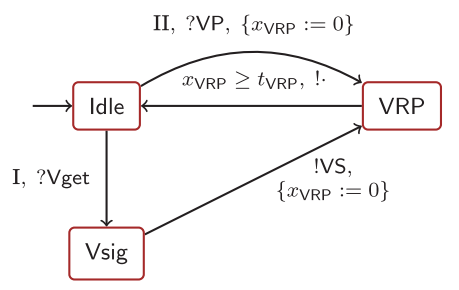

(b) VRP component

Fig. 8. Components of the VVIR pacemaker model.

Recall that, in the open-loop variant of the VVIR design, QTIs are extracted from offline ECG data and not generated from the heart model.

\subsection{VVIR Pacemaker Model}

In this section, we introduce the pacemaker model, the blending algorithm, and the estimation of QT-RR laws for rate adaptation.

VVIR Pacemaker. The PTIOA network of the VVIR pacemaker is shown in Figure 8. The model is a simplified version of the dual-chamber pacemaker model of Jiang et al. (2012), from which we only retain the components responsible for sensing and pacing the ventricle. We remark that, in the pacemaker network, delays are deterministic. The Lower Rate Interval (LRI) component ensures a given lower HR, which in our case corresponds to the adaptive rate. The adaptive pacing period is denoted with $t_{\mathrm{AR}}$ and is updated at regular intervals by the sensor blending algorithm (see Section 4.3.1), typically every 2.5 seconds. If there is no sensed ventricular event (represented by the synchronization on action VS) before $t_{A R}$ expires, the component paces the ventricle, which is implemented by output action !VP.

On the other hand, the Ventricular Refractory Period (VRP) component is responsible for the sensing of intrinsic ventricular events. These are generated by the heart network through action Vget. After a Vget is detected, the VRP component notifies the LRI component of the sensed impulse by sending a VS action. After a sensed or paced ventricular event, corresponding respectively to firing the edge Vsig $\rightarrow$ VRP and Idle $\rightarrow$ VRP, the pacemaker enters a refractory period (location VRP), during which sensing is disabled; i.e., no synchronization on Vget is possible. This mimics the natural refractory period of the ventricles, and thus allows for filtering out sensing noise. After the refractory period $t_{\mathrm{VRP}}$ has elapsed, the component returns to the Idle location where sensing is reactivated.

4.3.1 Blending Algorithm. The blending algorithm combines data from the QT and accelerometer sensors in order to change the adaptive pacing rate $t_{A R}$ such that the pacemaker mimics the behavior of a healthy conduction system in terms of mental and physical stress. The algorithm accounts for the fact that the accelerometer quickly reacts at the onset of exercise, but lacks precision in the longer run. On the other hand, the QT sensor gives very accurate and specific HR predictions, but has a slower response to exercise (Lau et al. 2007).

We propose a blending algorithm that generalizes the rate adaptation approaches presented in Lau et al. (2007). Let $t_{A R}^{\mathrm{QT}}$ and $t_{\mathrm{AR}}^{\mathrm{ACC}}$ be the adaptive pacing intervals computed by the $\mathrm{QT}$ and the accelerometer sensors, respectively, as detailed in Sections 4.3.2 and 4.3.3. Let $\mathrm{RR}_{\text {rest }}$ denote the heart period at rest. For the computation of the adaptive pacing interval $t_{\mathrm{AR}}$, our algorithm distinguishes four cases: 


$$
\mathrm{t}_{\mathrm{AR}}= \begin{cases}\mathrm{RR}_{\text {rest }} & \text { if } \mathrm{t}_{\mathrm{AR}}^{\mathrm{QT}} \geq \mathrm{RR}_{\text {rest }} \wedge \mathrm{t}_{\mathrm{AR}}^{\mathrm{ACC}} \geq \mathrm{R}_{\text {rest }} \\ \mathrm{t}_{\mathrm{AR}}^{\mathrm{QT}} & \text { if } \mathrm{t}_{\mathrm{AR}}^{\mathrm{QT}}<\mathrm{RR}_{\text {rest }} \wedge \mathrm{t}_{\mathrm{AR}}^{\mathrm{ACC}}<\mathrm{RR}_{\text {rest }} \\ \max \left(\mathrm{t}_{\mathrm{AR}}^{\mathrm{QT}}, \mathrm{ADL}\right) & \text { if } \mathrm{t}_{\mathrm{AR}}^{\mathrm{QT}}<\mathrm{RR}_{\text {rest }} \wedge \mathrm{t}_{\mathrm{AR}}^{\mathrm{ACC}} \geq \mathrm{RR}_{\text {rest }} \\ w(\Delta t) \mathrm{t}_{\mathrm{AR}}^{\mathrm{ACC}}+(1-w(\Delta t)) \mathrm{RR}_{\text {rest }} & \text { if } \mathrm{t}_{\mathrm{AR}}^{\mathrm{QT}} \geq \mathrm{R}_{\text {rest }} \wedge \mathrm{t}_{\mathrm{AR}}^{\mathrm{ACC}}<\mathrm{R}_{\text {rest }} .\end{cases}
$$

Case (i) corresponds to rest conditions, i.e., when none of the sensors indicate activity. Case (ii) describes sustained activity, i.e., when both sensors indicate activity, in which case $t_{A R}$ is updated to the pacing period suggested by the QT sensor. Case (iii) describes either mental or isometric activity, and occurs when only the QT sensor suggests activity. Also in this case, $\mathrm{t}_{\mathrm{AR}}$ is updated to $\mathrm{t}_{\mathrm{AR}}^{\mathrm{QT}}$ but the pacing period is limited by parameter $\mathrm{ADL}=0.66 \mathrm{~s}$ (activities of daily living) to avoid excessive pacing (Lau et al. 2007).

In case (iv), only the accelerometer detects activity, indicating either an early stage of activity (to which the QTI has not yet adapted) or a false detection. To reflect this duality, the blending algorithm opens a time window during which $t_{A R}$ is set to a weighted combination of $t_{A R}^{A C C}$ and $\mathrm{RR}_{\text {rest }}$, where the contribution of the accelerometer gradually decreases according to the parameter $w(\Delta t)$, defined as follows:

$$
w(\Delta t)= \begin{cases}-\frac{w_{0}}{t_{\max }} \Delta t+w_{0} & \text { if } \Delta t<t_{\text {max }} \\ 0 & \text { if } \Delta t \geq t_{\text {max }}\end{cases}
$$

where $w_{0}$ is the initial weight, $\Delta t$ indicates the time elapsed (in seconds) since case (iv) was entered, and $t_{\max }$ is the maximum time window. Following the blending algorithms reported in Lau et al. (2007), we set $w_{0}=0.8$ and $t_{\max }=60$.

Importantly, our algorithm can be easily adapted to reproduce the sensor blending algorithms of commercially available rate-adaptive pacemakers (Lau et al. 2007).

4.3.2 QT Sensor. In this section, we discuss methods to effectively compute such QT-RR laws, that is, to estimate a function $f$ such that

$$
\mathrm{QT} \approx f(\mathrm{RR}) .
$$

This equation is used within the blending algorithm to update the adaptive pacing rate period $t_{A R}^{Q T}$ from QTI values. Below, we illustrate the methods used to derive $f$ for patient-specific models, and for virtual population models.

Patient-Specific QT-RR Laws. Several attempts have been made to formalize the QT-RR relationship, even if experimental and statistical evidence shows that no single law exists that optimally fits every patient (Malik et al. 2002). We perform an in-depth analysis and comparison of five established mathematical models for describing patient-specific QT-RR relationship, namely:

(1) Linear model: QT $=a_{0}+a_{1} \mathrm{RR}$

(2) Quadratic model: $\mathrm{QT}=a_{0}+a_{1} \sqrt{\mathrm{RR}}$

(3) Cubic model: QT $=a_{0}+a_{1} \sqrt[3]{\mathrm{RR}}$

(4) Hyperbolic model: QT $=a_{0}+\frac{a_{1}}{\mathrm{RR}}$

(5) Exponential model: QT $=a_{0}-a_{1} \exp \left(-a_{2} \mathrm{RR}\right)$

We further investigate, for the first time in this context, the application of Multigene Symbolic Regression (MSR) (Searson 2015). MSR consists in the automatic computation of a regression law from a set of basis functions, and thus it notably reduces the need for trial-and-error fitting that results from fixed choices of regression functions. We employ the MSR algorithm of Searson (2015) that crucially supports multiobjective optimization to evaluate the tradeoff between the goodness 
Subject 1

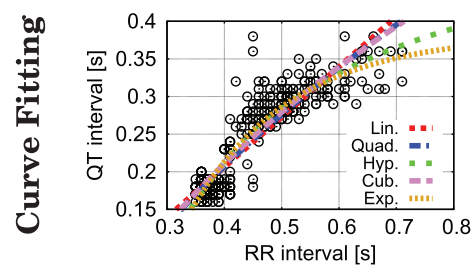

(a)

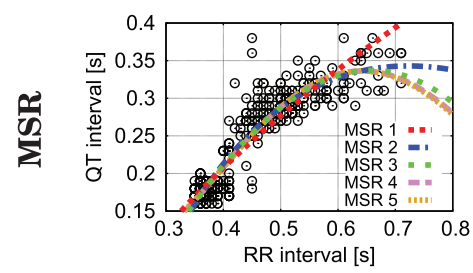

(d)
Subject 2

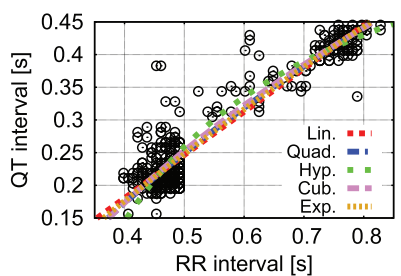

(b)

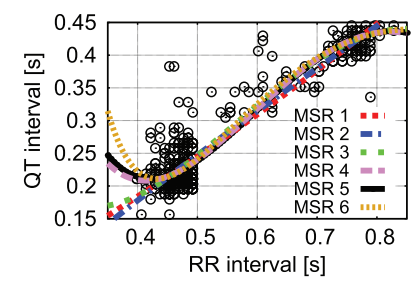

(e)
Subject 3

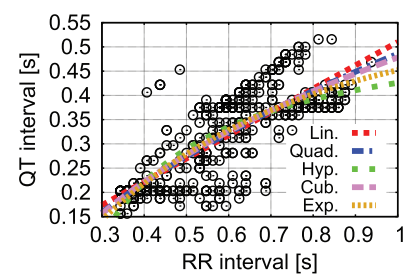

(c)

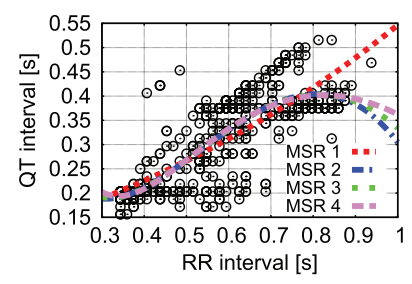

(f)

Fig. 9. Estimation of QT-RR laws for the three subjects of our study, and comparison between classical curve fitting (top row) and multigene symbolic regression (bottom row). For the latter, we show the Pareto-optimal models found.

of fit and the expressional complexity of a fitting function. In this way, MSR allows for a Paretooriented analysis of different response functions. To ensure a fair comparison, MSR was evaluated by choosing models (1) through (5), $\{+, \cdot,-, /, \sqrt{ }, \sqrt[3]{,}, \exp \}$, as the set of basis functions.

Curve-fitting results (plotted in Figure 9) evidence that (1) QT-RR laws are highly patient specific; (2) MSR produces models with superior accuracy, outperforming the above classical mathematical laws; (3) among models (1) through (5), the exponential law yields overall the best fitting scores; and (4) unlike the MSR models, the hyperbolic and exponential laws are characterized by good extrapolation accuracy-i.e., they provide satisfactory predictions also for points outside the training dataset.

In general, MSR represents an effective alternative to the current practice of choosing a fixed a priori mathematical model. Indeed, the "one fits all" approach can lead to inaccurate computation of the pacing rate period $t_{A R}^{\mathrm{QT}}$ computed by the QT sensor. Nevertheless, relative to our input data, the exponential law showed a satisfactory overall performance. Further details on MSR and fitting results are in Supplementary Material, Section 3.2.

Population-Level QT-RR Laws. As we just showed, the QT-RR relationship is patient specific, and thus, for the case of virtual population models, we have to resort to different, more general techniques.

To this purpose, we follow the work of Poore and Mann (1991), where a general mathematical law for computing the adaptive pacing rate is proposed that does not require patient-specific information, but only minimum and maximum values for the $\mathrm{RR}$ and the physiological sensor.

Let $\mathrm{QT}_{\max }\left(\mathrm{QT}_{\min }\right)$ and $\mathrm{RR}_{\max }\left(\mathrm{RR}_{\min }\right)$ be the maximum (minimum) $\mathrm{QT}$ and $\mathrm{RR}$ interval observed for the pool of patients considered. This law assumes linear updates from the minimum to the maximum values, i.e., a linear model with parameters

$$
a=\frac{\mathrm{QT}_{\max }-\mathrm{QT}_{\min }}{\mathrm{RR}_{\max }-\mathrm{RR}_{\min }} \quad \text { and } \quad b=\mathrm{QT}_{\min }-a \cdot \mathrm{RR}_{\min } .
$$




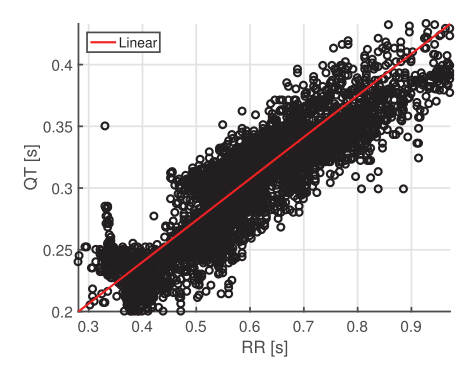

Fig. 10. Estimation of linear QT-RR law for the virtual population.

The estimated linear model for our virtual population is shown in Figure 10. Compared to the results of patient-specific estimation, we observe that, for each RR interval, observed QTIs have much higher variability, and that the overall trend is more markedly linear. We remark that this law can be also used for patients affected by permanent atrial impairment, i.e., whose ECG contains no useful QT-RR pairs for the above patient-specific estimation, since it relies on maximal and minimal HR that can be estimated from population-wide models, as per Equation (5) below.

4.3.3 Accelerometer Sensor. We process three-axial accelerometer signals in order to detect the physical activity of the patient. Specifically, we derive a real-time approximation of the patient Metabolic Equivalent of Task (MET), an index of physical activity intensity defined as the ratio of the work metabolic rate to the resting metabolic rate. Thus, a MET value of 1 indicates resting, and typically, light physical activities have an associated MET value $<3$, while MET values for intense activities are $>6$.

The MET computation is achieved by a decision tree model, estimated by Ohkawara et al. (2011) from the statistical analysis of patient data, and its value is updated at runtime by inspecting a time window of 5 seconds. Let $X$ be the mean value of the accelerometer signal during the time window, and $\hat{X}$ be the mean of the corresponding filtered signal, obtained after removing the baseline drift. The model distinguishes among sedentary activity, light activity, and heavy activity, and derives the MET based on $X$ and $\hat{X}$ as follows:

$$
\operatorname{MET}=\left\{\begin{array}{lrr}
1 & \text { if } \hat{X}<k_{0} & \text { (sedentary activity) } \\
a_{0}+a_{1} \cdot \hat{X} \text { if } \frac{X}{\hat{X}} \geq k_{1} & \text { (light activity) } \\
b_{0}+b_{1} \cdot \hat{X} \text { otherwise } & \text { (heavy activity) }
\end{array}\right.
$$

where $k_{0}, k_{1}, a_{0}, a_{1}, b_{0}, b_{1}$ are the parameters estimated in Ohkawara et al. (2011).

Finally, the adaptive pacing period suggested by the accelerometer, $t_{A R}^{A C C}$, is derived using Wilkoff's patient-dependent chronotropic response model (Wilkoff et al. 1989), which computes, for a given MET value, the appropriate HR:

$$
\mathrm{HR}(\mathrm{MET})=\frac{\left(220-\text { age }-\mathrm{HR}_{\text {rest }}\right)(\mathrm{MET}-1)}{\mathrm{MET}_{\max }-1}+\mathrm{HR}_{\text {rest }} \text { and } \mathrm{t}_{\mathrm{AR}}^{\mathrm{ACC}}=\frac{60}{\mathrm{HR}(\mathrm{MET})},
$$

where age is the age of the patient, $\mathrm{HR}_{\text {rest }}$ is the patient's $\mathrm{HR}$ at rest, and $\mathrm{MET}_{\max }$ is the maximum MET value. Values of age and $\mathrm{HR}_{\text {rest }}$ for our virtual patients are given in Table 1 of Section 4.1. Note that the minimum HR is obtained at minimum MET $(M E T=1)$ and is equal to $\mathrm{HR}_{\text {rest }}$, while for $\mathrm{MET}=\mathrm{MET}_{\max }$ we recover the well-known equation for maximum HR: $220-$ age. Alternative laws for the maximum HR have been proposed (Tanaka et al. 2001) but are outside the scope of our study. 


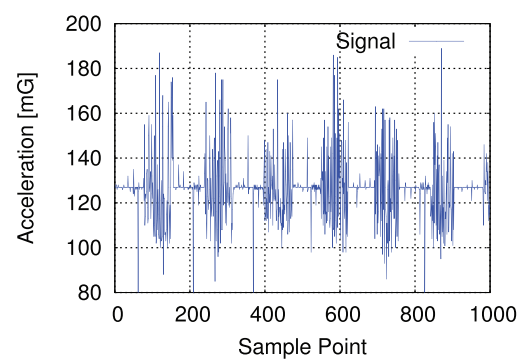

(a) Segment of accelerometer signal

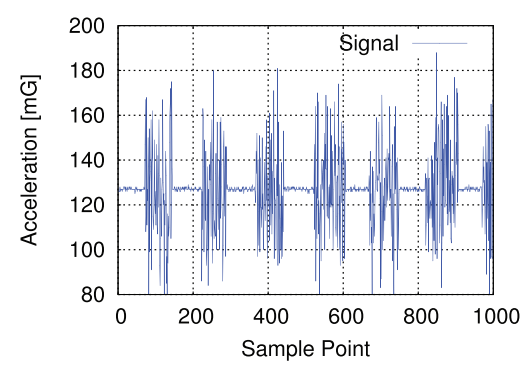

(b) Corresponding synthetic signal

Fig. 11. Example of the results produced by our algorithm for the generation of patient-specific, activitydependent, synthetic accelerometer signals.

\subsection{Generation of Synthetic Accelerometer Signals}

We introduce a new method for the generation of synthetic, three-axial accelerometer signals that are either patient specific (i.e., they resemble a given input signal) or activity dependent (i.e., they describe a specific physical activity, e.g., walking or running). Our method is based on a Poisson process model, whose events represent the onset of activity in the generated signal, e.g., climbing stairs.

Let $\bar{t}$ be a time bound and $T_{1}(\beta), \ldots, T_{N}(\beta)$ be random variables describing the absolute arrival time up to time $\bar{t}$ of $N$ activity events, such that for $i=1, \ldots, N, T_{i}(\beta)-T_{i-1}(\beta)$ is exponentially distributed with mean arrival time $\beta .{ }^{11}$ The durations of the activity segments are modeled as independent and identically distributed Gaussian random variables $L_{1}(\mu, \sigma), \ldots, L_{N}(\mu, \sigma)$, where $\mu$ and $\sigma$ are, respectively, the mean and standard deviation of the distribution. The typical shape of the accelerometer signal during these segments is reproduced by means of a white Gaussian noise generator (Wang et al. 2011). For each time point $t \in[0, \bar{t}]$ and interval $\left[t_{1}, t_{2}\right) \subseteq[0, \bar{t}]$, we define the white Gaussian noise generator $W G N_{t_{1}}^{t_{2}}\left(t ; \sigma_{p}\right)$ as a random variable that is normally distributed with zero mean and standard deviation equal to $\sigma_{p}$ if $t \in\left[t_{1}, t_{2}\right)$, and identically zero otherwise. The synthetic accelerometer signal at time $t$ is hence a random variable defined as

$$
\operatorname{synthAcc}\left(t ; \beta, \mu, \sigma, \sigma_{p}\right)=\sum_{i=1}^{N} W G N_{T_{i}(\beta)}^{T_{i}(\beta)+L_{i}(\mu, \sigma)}\left(t ; \sigma_{p}\right) \text {. }
$$

We finally add noise to the signal in order to mimic background noise effects. Parameters $\beta, \mu$, $\sigma$, and $\sigma_{p}$ are among the most used features for accelerometer signal processing (Chernbumroong et al. 2011) and can be automatically estimated from accelerometer recordings, or tuned to reproduce a prescribed, activity-dependent morphology. For the former, we have implemented a detection algorithm able to identify the regions of interest in the signal, and extract the relevant statistical features from it. An example of synthetic accelerometer signal is shown in Figure 11, using as input accelerometer data taken from Anguita et al. (2013). We can observe that the key features of the input signal are preserved by our algorithm and are clearly visible in the generated synthetic signal.

Validation. To validate our algorithm, we need to show that the input accelerometer signal and the corresponding synthetic signals are consistent with respect to the decision tree model used for the accelerometer; that is, the two signals must be classified to the same activity level (sedentary,

\footnotetext{
${ }^{11}$ We assume $T_{0}(\beta)=0$.
} 


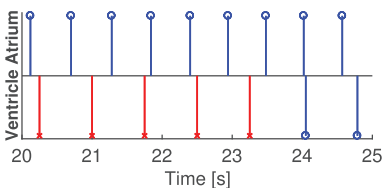

(a) VVI stem plot.

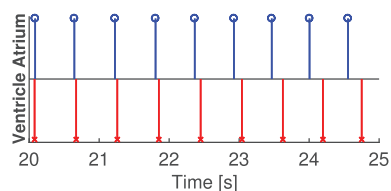

(b) VVIR stem plot

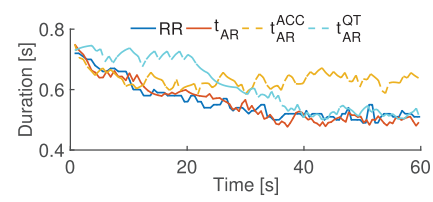

(c) Sensors comparison.

Fig. 12. Results of model simulation. Left and center figures depict the stem plots of a 5-second simulation (upper half, atrial activity; lower half, ventricular). Blue stems indicate intrinsic atrial impulses, or atrial impulses conducted in the ventricle; red stems indicate paced ventricular beats. Right: pacing period of different rate-adaptive sensors: blended (red), accelerometer (yellow), and QT based (aqua), compared to the ideal demand (blue).

light, or heavy activity). We are also interested in how the predicted metabolic activity differs between the two.

To this end, we consider 100 recordings from the training set of Anguita et al. (2013) and generate, for each, 100 synthetic signals. Our synthetic signal generator produced a satisfactory $93 \%$ of correct classifications, with a mean deviation of only 0.17 MET in the predicted metabolic activity.

\section{RESULTS}

In Section 5.1, we analyze the behavior of the VVIR pacemaker along a single simulation, and how it compares with alternative pacemaker designs. In Section 5.2, we illustrate the verification results obtained through statistical model checking.

Unless otherwise stated, the following experiments assume a condition of AV block and AF (modeled as per Section 4.2), which is typically addressed by VVIR pacing (Iaizzo 2009, Fig. 30.7-8).

Implementation. The COSMOS statistical model checker works by executing efficient $\mathrm{C}++$ code generated from Stochastic Petri Net (SPN) models. Therefore, we apply existing methods (Barbot et al. 2016) to translate the heart-pacemaker PTIOA network into an SPN. The blending and signal processing algorithms described in Sections 4.3.1 to 4.4 are implemented as $\mathrm{C}++$ code, called by COSMOS during execution. Note that these algorithms have only the effect of updating variable values of the PTIOA network. An implementation is available at www.veriware.org/heart_pm methods.php\#heartverify.

\subsection{Simulation and Comparison with Fixed-Rate Pacemaker}

In this section, we compare our VVIR pacemaker design with its fixed-rate variant (called VVI) during sustained activity, and further compare the dual-sensor blending algorithm with the corresponding single-sensor variants, i.e., obtained by disabling one of the two sensors. For a deeper look at the behavior of the VVIR pacemaker, here we consider a single simulation trace, parameterized with an extract of the ECG and accelerometer data from subject 1 during activity (see Section 4.1), and choose an exponential QT-RR law in the blending algorithm.

Given that subject 1 has a healthy ECG, we use the corresponding sequence of RR intervals to construct the ideal rate demand curve. Recall that in our model, the ideal rate demand is reflected by the firing rate of the SA node (see Section 4.2.3), which propagates impulses into the atrium. Therefore, to obtain a clear illustration of the atrial activity, only for this experiment, we assume no AF (only AV block).

Analysis of Pacing Modes. The results are summarized in Figures 12(a) and 12(b), which give a detailed view of the atrial and ventricular events during a 5-second simulation. We observe that, 
in the VVI mode, the fixed ventricular rate imposed by the pacemaker is not commensurate to the atrial activity. There are only a few impulses successfully conducted from the atrium to the ventricle, which happens at regular intervals in the Wenckebach AV block. This behavior does not occur in the VVIR mode, where the adaptive pacing rate ensures that the ventricular rhythm follows the ideal demand through increased pacemaker activity.

Analysis of Blending Algorithms. In Figure 12(c), we compare the pacing rates computed by our blending algorithm under exercise with the single-sensor pacing rates. We observe that, if we consider only the accelerometer, the rate-adaptive pacemaker reacts appropriately to the onset of exercise (visible by the decreased RR duration) and sets an adequate pacing rate for the first $\approx 20$ seconds, after which it cannot account for the increased rate demand. On the other hand, the QT sensor alone fails to detect the onset of activity in a timely manner, but after the first $\approx 25$ seconds provides more and more accurate predictions. Our blending algorithm takes advantage of both sensors: it successfully detects the onset of patient activity and predicts the right chronotropic response in the longer run, with the resulting adaptive rate closely following the ideal demand at any time.

\subsection{Statistical Model Checking Experiments}

We employ SMC to provide formal and quantitative guarantees on the behavior of the VVIR pacemaker. Our study involves two broad kinds of analyses, in order to investigate (1) effects of pacemaker malfunctions on cardiac dynamics and (2) the adequacy of pacing treatment under varying physiological conditions.

The experiments of Sections 5.2.2 and 5.2.3 belong to the first kind of analysis. In the former experiment, we investigate phenomena of sensor-induced endless-loop tachycardia, comparing closed- and open-loop designs. In the latter experiment, we analyze how the heart rate regularity is affected by a faulty pacemaker lead that causes oversensing. This defect is typically random and thus can be meaningfully investigated through SMC.

The experiments of Sections 5.2.4 and 5.2.5 belong to the second kind of analysis. In the former experiment, we simulate exercise curves and stress tests in order to assess which QT-RR law provides the most adequate rate at every activity stage. In the latter experiment, we analyze how the pacemaker responds to different conditions of the cardiac conduction system, namely, at different degrees of AV block.

Where not otherwise specified, the following analyses are obtained by instantiating the QT sensor of the rate-adaptive algorithm with the exponential law. For SMC, we only consider timebounded HASL properties. ${ }^{12}$

\subsubsection{Verification Properties.}

Absence of Induced Tachycardia. This HASL property evaluates the probability that, in resting conditions, the pacemaker does not wrongly switch to activity mode, thus inducing an excessive HR (tachycardia). The corresponding LHA (illustrated in Supplementary Material, Section 5) is designed to count, through variable nOKs, the number of adequate rate updates, i.e., inducing an effective HR that does not exceed the HR at rest of the subject. Variable nUpd keeps track of the total number of rate updates. The HASL formula is defined as

$$
\phi_{\mathrm{NO} \_\mathrm{TC}}=\mathbb{E}\left[\operatorname{LAST}\left(\frac{\mathrm{nOKs}}{\mathrm{nUpd}}\right)\right],
$$

\footnotetext{
${ }^{12}$ These are characterized by an LHA with a global clock $t$ and unique final state whose incoming edges are autonomous and have guard $g=t \geq \operatorname{Tmax}$, where Tmax is the time bound.
} 
corresponding to the expectation of the final value of the ratio of adequate updates. Since the adequacy/nonadequacy of each update forms a Bernoulli process, $\phi_{\mathrm{NO}} \mathrm{TC}$ actually estimates the probability that the rate is updated correctly, in a way that it does not induce tachycardia.

PDF of Fraction of Paced Beats. We consider the HASL property $\phi_{\mathrm{PDF}(\mathrm{VP})}$ introduced in Example 3.1. The property evaluates the PDF of the fraction of paced beats, or, simply put, how much artificial pacing is needed to ensure a safe heart rhythm.

Heart Rate Regularity. We introduce a measure of HR regularity for a patient under pacing conditions, defined as the average deviation between consecutive RR intervals. Let $n$ be the number of beats along a given execution, and $R R_{i}$ be the $\mathrm{RR}$ associated to the $i$ th beat. We define the regularity of the path as

$$
\operatorname{Reg}=\frac{1}{n-1} \sum_{i=1}^{n-1}\left|R R_{i+1}-R R_{i}\right| .
$$

Low Reg values are indicative of paths where the RRs change smoothly, whereas high Reg values indicate abrupt HR changes, potentially introducing excessive stress on the patient's cardiac tissue. Due to the intrinsic HR variability, a value of zero for Reg is impossible to achieve.

In this regard, we can compute a baseline level for Reg, corresponding to the regularity of a perfectly healthy subject in a particular activity stage. This can be obtained by assuming that the $\mathrm{RR}$ delays of a healthy subject in a particular stage of activity are normally distributed (as done in Lian et al. (2010)) and is defined by

$$
\operatorname{Reg}^{*}=2 \sqrt{\frac{\sigma^{2}}{\pi}}
$$

where $\sigma$ is the standard deviation of the estimated Gaussian distribution for RR intervals at the given activity stage. The full derivation of Equation (8) is in Supplementary Material, Section 5.

The LHA for computing Reg over a simulation path is illustrated in Supplementary Material, Section 5. It contains a variable $\operatorname{Reg}_{\Sigma}$ for storing the cumulative sum of the deviations between consecutive RRs, and a variable Nbeats, tracing the number of ventricular beats. The HASL formula is

$$
\phi_{\text {Reg }}=\mathbb{E}\left[\operatorname{LAST}\left(\frac{\operatorname{Reg}_{\Sigma}}{\text { Nbeats }}\right)\right] .
$$

5.2.2 Endless-Loop Tachycardia: Open versus Closed Loop. We analyze phenomena of pacemaker-mediated tachycardia (PMT), also called endless-loop tachycardia, and compare the accuracy of the open- and closed-loop VVIR architectures in reproducing this malfunction. PMT is typical of (dual-chamber) DDD pacemakers (Monteil et al. 2015) but has been found to occur also in VVIR pacemakers (see, e.g., Bohm et al. (2010)). In this case, the event is triggered by a sensor malfunction causing the pacemaker to wrongly detect activity, thus inducing a self-sustained increase of the ventricular pacing rate, as illustrated in Figure 13.

To intentionally induce PMT in our model, we consider a naïve blending algorithm where crosschecking of multiple sensors is disabled and thus, any of the two sensors can set the pacing rate as it predicts. The PMT episode is initiated by simulating a fault in the accelerometer sensor that detects activity when the subject is actually at rest. This results in a wrongly increased pacing rate and, as a side effect, in a shortening of the QTI (Ahnve and Vallin 1982). In turn, shorter QTIs are translated by the QT sensor into higher pacing frequencies, leading to endless-loop tachycardia.

We verify property $\phi_{\mathrm{NO} \_\mathrm{TC}}($ see Equation $(7))$ to estimate the probability that, after an induced sensor malfunctioning of 30 seconds, the pacemaker does not induce tachycardia in the following 90 seconds. Detailed experimental setup and numerical results are reported in Supplementary Material, Section 5. In a nutshell, we obtain that in the closed-loop architecture, PMT is from $18 \%$ to 


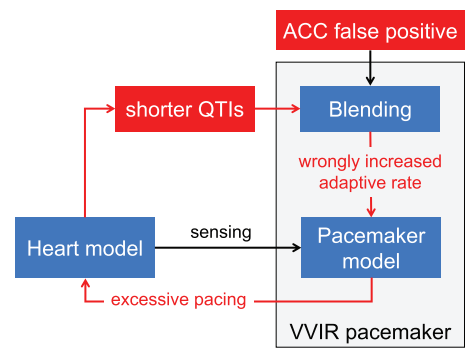

Fig. 13. Feedback behavior of sensor-induced tachycardia.

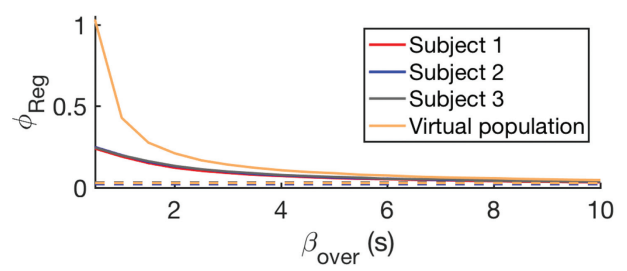

(a) Rest

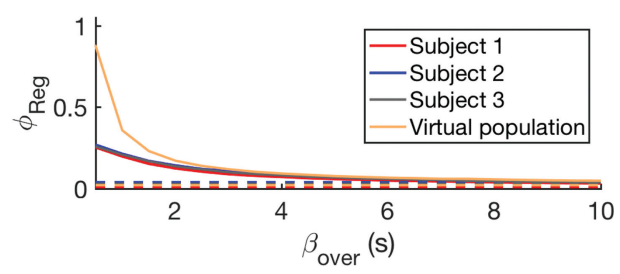

(b) Activity

Fig. 14. HR regularity ( $\phi_{\mathrm{Reg}}, \mathrm{y}$-axis) at different oversensing rates ( $\beta_{\text {over }}, \mathrm{x}$-axis). Mean and $99 \%$ confidence intervals for $\phi_{\text {Reg }}$ are indicated with lines and shaded areas, respectively. Dashed lines represent the corresponding ideal regularity values (Reg*, see Equation (8)). For SMC, we use 1,000 simulations for each subject.

$59 \%$ more likely to happen than in the open-loop case across the three virtual patients, for which tachycardia occurs with relatively low probability (ranging from $1 \%$ to $30 \%$ ).

Therefore, SMC results confirm that the feedback-loop nature of PMT can be only reproduced in the closed-loop model and cannot be well captured by the open-loop model, since this uses offline data that does not reflect the runtime activity of the pacemaker. We remark, however, that when using the complete blending algorithm of Section 4.3.1, PMT is terminated.

5.2.3 Regularity under Oversensing. We analyze how oversensing, i.e., the misinterpretation of artifacts or other waves as $\mathrm{R}$ peaks by the pacemaker, affects the HR regularity (see Equation (5.2.1)). The occurrence of an oversensing event is modeled as an exponentially distributed variable, with mean waiting time given by the parameter $\beta_{\text {over }}$, ranging in the interval $[0.5,10]$ seconds. We evaluate regularity in both resting and activity conditions through SMC of the HASL formula $\phi_{\text {Reg }}$ (see Equation (9)).

Results for $\phi_{\text {Reg }}$ are illustrated in Figure 14. Recall that smaller $\phi_{\text {Reg values imply a more regular }}$ heart rhythm. As expected, the worst regularity is registered when severe oversensing is in action, and this monotonically improves as the rate of misdetected beats decreases.

We obtain similar results among the three patient-specific models and between resting and activity conditions, suggesting that the pacemaker is able to maintain good levels of regularity independently of the activity stage and the differences intrinsic to the three subjects.

For the virtual population model, this analysis yields considerably higher values of $\phi_{\text {Reg }}$ (less regular HR) for high values of oversensing (i.e., low values of $\beta_{\text {over }}$ ). This is explained by the higher width of the parameter distributions estimated by the population-wide approach. Note, however, that values of $\phi_{\text {Reg }}$ comparable to those obtained for the patient-specific approach are restored for high values of $\beta_{\text {over }}$. 
We further observe that the obtained confidence intervals for $\phi_{\text {Reg }}$ are very narrow, meaning that SMC results are precise and, more importantly, that the regularity measure is robust with respect to the intrinsic heart variability and the random occurrence of oversensing events.

5.2.4 Adequacy of Pacing Rate during Exercise. We evaluate how the HR under AV block and AF is corrected by the rate-adaptive pacemaker during exercise, and in particular, how the effective HR induced by the pacemaker fits the required ideal rate demand (ID). Recall that, in our model, the ID is set by updating the firing period of the SA node (see Section 4.2.3).

We consider three experiments, respectively describing

(1) a vigorous, 20-minute exercise consisting of four activity stages: activity onset, sustained activity, decay, and rest;

(2) a more intense, 10-minute exercise consisting of three stages: activity onset, sustained activity, and decay; and

(3) the Bruce protocol (Candinas et al. 1997), one of the most common clinical stress tests. It is composed of seven activity stages performed at increasing intensity, with a duration of 3 minutes each. The ID at each stage is described in MET, from which we derive the corresponding ideal HR by using Equation (5) for each virtual patient (see Supplementary Material, Section 6 for the ideal HR of each patient).

Regarding accelerometer data, we generate synthetic signals using parameters estimated from recordings at rest (for rest and decay stages) and during activity (for onset and sustained activity stages). As explained in Section 4.1, we use accelerometer data from Anguita et al. (2013) for subject 2 and for the virtual population.

Results are shown in Figure 15 and compare the behavior of different QT-RR laws in the blending algorithm: linear model, exponential model, and lowest mean-square error MSR model. We generally observe that the effective HR qualitatively and quantitatively agrees with the ID, demonstrating that the blending algorithm is able to provide an adequate heart behavior during exercise.

In particular, for the Bruce protocol, the rate-adaptive algorithm achieves an almost ideal behavior, accounting for the sudden ID changes in a timely manner when transitioning between activity stages, and successfully maintaining the effective HR constant during each stage. The only exception is the linear model for subject 1, which underestimates the ID during stages 4 through 6 . The cause of this behavior is to be found in the decreased fitting performance of the linear model for the corresponding range of RR values (see Figure 9(a)), even though the discrepancy between the ID and effective rate is never above $20 \mathrm{BPM}$.

Noteworthy are the narrow confidence intervals in the patient-specific experiments (subjects 1-3), obtained with just 100 simulations, independently of the subject or the used QT-RR law. This proves the robustness of our pacemaker design, able to mitigate the intrinsic stochasticity of the heart model.

Results obtained for the virtual population demonstrate that, also in this case, the rate-adaptive pacemaker is able to follow the overall trend of the exercise curves, with a very similar performance to the patient-specific experiments. At sustained activity rates, when the pacing rate is mainly determined by the QT sensor, we notice an increased width of the confidence interval, which mirrors the increased variability of the QTIs estimated from multiple patients.

Further note that the quick initial HR increase, as well as the quick decrease at the decay stage (for exercise curves 1 and 2), is basically independent from the QT-RR law used in the QT sensor and of the virtual patient. This is in fact mostly driven by the accelerometer sensor that successfully detects the onset/offset of activity. 


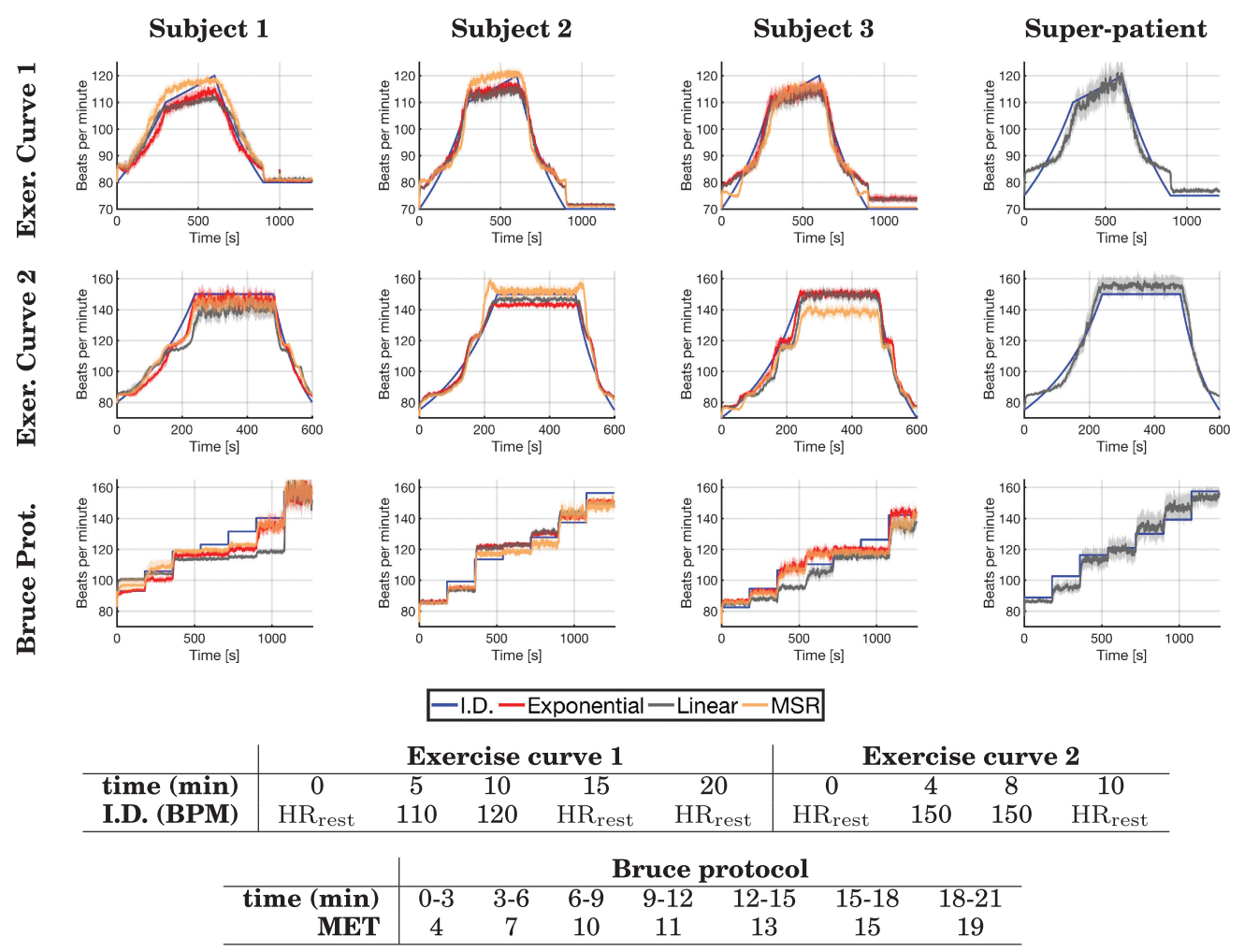

Fig. 15. HR during simulated exercise curves, obtained through 100 simulations for each subject. The ideal rate demand (ID, blue) is compared to the HR under different QT-RR laws for the blending algorithm: exponential, linear, and best-fitting MSR model. Shaded areas indicate $99 \%$ confidence intervals. Tables describe the different stages of each test.

5.2.5 AV Block Degree. We perform a parametric analysis of AV_Vt, i.e., the AV node depolarization threshold that, as explained in Section 4.2, can be tuned to reproduce AV block conditions, such that higher AV_Vt values imply worse heart conduction. In particular, we let AV_Vt deviate from its default (healthy) value of $-4.0 \mathrm{mV}$ (Barbot et al. 2015a) by an exponentially distributed random variable with mean $\beta_{v t}$. We consider property $\phi_{\mathrm{PDF}(\mathrm{VP})}$, defined in Example 3.1, to evaluate the probability density function of the fraction of paced beats, with $\beta_{v t}$ spanning from 0.1 (normal AV conduction) to 2.5 (severe AV block).

The heat-maps in Figure 16 depict, for each subject and for both resting and sustained activity conditions, how the distribution of the fraction of paced beats is affected by parameter $\beta_{v t}$. We observe that as the condition of the conduction system worsens, the number of paced beats increases, demonstrating that the pacemaker can well adapt to different degrees of AV block. This increase slowly saturates around the value of 0.9 . This indicates that the coexistence of $\mathrm{AF}$ and severe AV block in a subject makes the pacemaker almost essential, with only a small percentage of atrial impulses propagating into the ventricles. At high $\beta_{v t}$ values, we further notice a substantial decrease of the width of the distributions (redder area, implying a higher probability density). This suggests a scenario where heart cycles are primarily initiated by the pacemaker, effectively reducing the intrinsic variability of the patient (diseased) heart dynamics. 


\section{Subject 1}
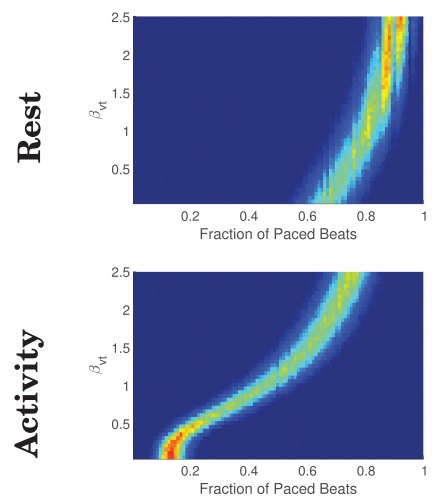

Subject 2
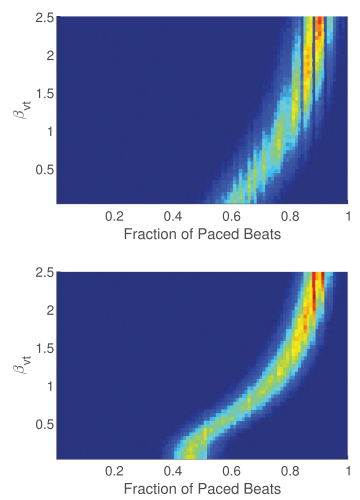

Subject 3
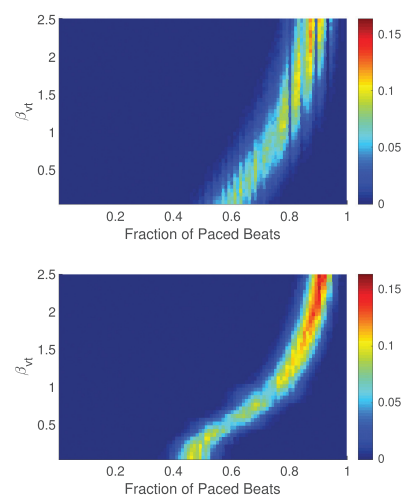

Fig. 16. Probability density function of the fraction of paced beats (x-axis) under different degrees of AV block severity, obtained by increasing the AV node threshold potential by parameter $\beta_{v t}$ ( $y$-axis). Colors indicate the SMC results for formula $\phi_{\mathrm{PDF}(\mathrm{VP})}$ (see Example 3.1), evaluated for each value of $\beta_{v t}$ using a total of 50,000 simulations.

For the virtual population, we report a fraction of paced beats always greater than 0.9 , independently of the AV block degree and of the activity stage. This suggests that the QT-RR law implemented for the virtual population is more conservative, causing the device to pace the heart even when only $\mathrm{AF}$ and no AV block is present.

\section{CONCLUSION}

Safety assurance of rate-adaptive pacemakers demands rigorous verification methods able to account for the high specificity of the patient's electrophysiology, and how this changes under different levels of physical or mental stress. In this work, we addressed this challenge through the development of a formal, data-driven, and model-based approach to the automated, closed-loop verification of dual-sensor rate-adaptive pacemakers. Our approach uniquely combines methods for the personalization of heart models from patient data with the online generation of synthetic, model-based sensor data used for rate adaptation, seamlessly supporting patient-specific models for the analysis of personalized treatments and virtual population models targeted to safety verification. We performed extensive statistical model checking experiments to assess the device's behavior under a variety of conditions, leading to quantitative insights that would be difficult, if not impossible, to obtain with testing on real patients. By considerably reducing exercise testing, our techniques offer great potential to enhance the design and development process of rate-adaptive pacemakers, and to provide clinicians with appropriate and patient-specific treatment indications.

\section{REFERENCES}

Freddy M. Abi-Samra, Narendra Singh, Benjamin L. Rosin, Jerome V. Dwyer, Crystal D. Miller, and CLEAR Study Investigators. 2013. Effect of rate-adaptive pacing on performance and physiological parameters during activities of daily living in the elderly: Results from the CLEAR (cylos responds with physiologic rate changes during daily activities) study. Europace 15, 6 (2013), 849-856.

Staffan Ahnve and Hans Vallin. 1982. Influence of heart rate and inhibition of autonomic tone on the QT interval. Circulation 65, 3 (1982), 435-439.

Weiwei Ai, Nitish Patel, and Partha Roop. 2016. Requirements-centric closed-loop validation of implantable cardiac devices. In Design, Automation \& Test in Europe Conference \& Exhibition (DATE'16). IEEE, 846-849. 
Weiwei Ai, Nitish D. Patel, Partha S. Roop, Avinash Malik, Sidharta Andalam, Eugene Yip, Nathan Allen, and Mark L. Trew. 2018. A parametric computational model of the action potential of pacemaker cells. IEEE Transactions on Biomedical Engineering 65, 1 (2018), 123-130.

Paul Albrecht. 1983. ST Segment Characterization for Long Term Automated ECG Analysis. PhD Thesis. Massachusetts Institute of Technology, Department of Electrical Engineering and Computer Science.

Rajeev Alur. 1999. Timed automata. In International Conference on Computer Aided Verification. Springer, 8-22.

Francesco Amigoni, Alessandro Beda, and Nicola Gatti. 2006. Combining rate-adaptive cardiac pacing algorithms via multiagent negotiation. IEEE Transactions on Information Technology in Biomedicine 10, 1 (2006), 11-18.

Davide Anguita, Alessandro Ghio, Luca Oneto, Xavier Parra, and Jorge L. Reyes-Ortiz. 2013. A public domain dataset for human activity recognition using smartphones. In European Symposium on Artificial Neural Networks, Computational Intelligence and Machine Learning (ESANN'13).

Paolo Ballarini, Benoît Barbot, Marie Duflot, Serge Haddad, and Nihal Pekergin. 2015. HASL: A new approach for performance evaluation and model checking from concepts to experimentation. Performance Evaluation 90 (2015), 53-77.

Benoît Barbot, Marta Kwiatkowska, Alexandru Mereacre, and Nicola Paoletti. 2015a. Estimation and verification of hybrid heart models for personalised medical and wearable devices. In Computational Methods in Systems Biology (LNCS), Vol. 9308. Springer, 3-7.

Benoît Barbot, Marta Kwiatkowska, Alexandru Mereacre, and Nicola Paoletti. 2015b. Estimation and Verification of Hybrid Heart Models for Personalised Medical and Wearable Devices. Technical Report CS-RR-15-05. Department of Computer Science, University of Oxford.

Benoît Barbot, Marta Kwiatkowska, Alexandru Mereacre, and Nicola Paoletti. 2016. Building power consumption models from executable timed I/O automata specifications. In Hybrid Systems: Computation and Control (HSCC'16). ACM, 195204.

Chris Barker, Marta Kwiatkowska, Alexandru Mereacre, Nicola Paoletti, and Andrea Patanè. 2015. Hardware-in-theloop simulation and energy optimization of cardiac pacemakers. In IEEE Engineering in Medicine and Biology Society (EMBC'15). IEEE, 7188-7191.

Ezio Bartocci, Flavio Corradini, Maria Rita Di Berardini, Emilia Entcheva, Scott A. Smolka, and Radu Grosu. 2009. Modeling and simulation of cardiac tissue using hybrid i/o automata. Theoretical Computer Science 410, 33 (2009), 3149-3165.

Alan D. Bernstein, Jean-Claude Daubert, Ross D. Fletcher, David L. Hayes, Berndt Lüderitz, Dwight W. Reynolds, Mark H. Schoenfeld, and Richard Sutton. 2002. The revised NASPE/BPEG generic code for antibradycardia, adaptive-rate, and multisite pacing. Pacing and Clinical Electrophysiology 25, 2 (2002), 260-264.

Adam Bohm, Robert Kiss, Paul Dorian, and Arnold Pinter. 2010. Single-chamber, rate-responsive pacemaker-mediated tachycardia. Canadian fournal of Cardiology 26, 9 (2010), e340.

Reto Candinas, Markus Jakob, Thomas A. Buckingham, Heidy Mattmann, and F. Wolfgang Amann. 1997. Vibration, acceleration, gravitation, and movement: Activity controlled rate adaptive pacing during treadmill exercise testing and daily life activities. Pacing and Clinical Electrophysiology 20, 7 (1997), 1777-1786.

Taolue Chen, Marco Diciolla, Marta Kwiatkowska, and Alexandru Mereacre. 2014. Quantitative verification of implantable cardiac pacemakers over hybrid heart models. Information and Computation 236 (2014), 87-101.

Saisakul Chernbumroong, Anthony S. Atkins, and Hongnian Yu. 2011. Activity classification using a single wrist-worn accelerometer. In 2011 5th International Conference on Software, Knowledge Information, Industrial Management and Applications (SKIMA'11). IEEE, 1-6.

Patrick Davey and Jeffrey Bateman. 1999. Heart rate and catecholamine contribution to QT interval shortening on exercise. Clinical Cardiology 22, 8 (1999), 513-518.

Simonetta Dell'Orto, Paolo Valli, and Enrico Maria Greco. 2004. Sensors for rate responsive pacing. Indian Pacing and Electrophysiology fournal 4, 3 (2004), 137.

Simon Eberz, Nicola Paoletti, Marc Roeschlin, Andrea Patanè, Marta Kwiatkowska, and Ivan Martinovic. 2017. Broken hearted: How to attack ECG biometrics. In Network and Distributed System Security Symposium.

Kenneth A. Ellenbogen, Bruce L. Wilkoff, G. Neal Kay, Chu Pak Lau, and Angelo Auricchio. 2016. Clinical Cardiac Pacing, Defibrillation and Resynchronization Therapy. Elsevier Health Sciences.

Ary L. Goldberger, Luis A. N. Amaral, Leon Glass, Jeffrey M. Hausdorff, Plamen Ch. Ivanov, Roger G. Mark, Joseph E. Mietus, George B. Moody, Chung-Kang Peng, and H. Eugene Stanley. 2000. Physiobank, physiotoolkit, and physionet components of a new research resource for complex physiologic signals. Circulation 101, 23 (2000), e215-e220.

Artur Oliveira Gomes and Marcel Vinicius Medeiros Oliveira. 2009. Formal specification of a cardiac pacing system. In Formal Methods (FM'09). Springer, 692-707.

Radu Grosu, Gregory Batt, Flavio H. Fenton, James Glimm, Colas Le Guernic, Scott A. Smolka, and Ezio Bartocci. 2011. From cardiac cells to genetic regulatory networks. In Computer Aided Verification. Springer, 396-411.

John R. Hampton and David Adlam. 2013. The ECG in Practice. Elsevier Health Sciences.

Paul A. Iaizzo. 2009. Handbook of Cardiac Anatomy, Physiology, and Devices. Springer Science \& Business Media. 
Zhihao Jiang, Miroslav Pajic, Salar Moarref, Rajeev Alur, and Rahul Mangharam. 2012. Modeling and verification of a dual chamber implantable pacemaker. In Tools and Algorithms for the Construction and Analysis of Systems. Springer, 188-203.

Tamara G. Kolda, Robert Michael Lewis, and Virginia Torczon. 2003. Optimization by direct search: New perspectives on some classical and modern methods. SIAM Review 45, 3 (2003), 385-482.

Marta Kwiatkowska, Harriet Lea-Banks, Alexandru Mereacre, and Nicola Paoletti. 2014. Formal modelling and validation of rate-adaptive pacemakers. In 2014 IEEE International Conference on Healthcare Informatics (ICHI'14). IEEE, 23-32.

Marta Kwiatkowska, Alexandru Mereacre, Nicola Paoletti, and Andrea Patanè. 2015. Synthesising robust and optimal parameters for cardiac pacemakers using symbolic and evolutionary computation techniques. In Hybrid Systems and Biology (HSB’15) (LNCS/LNBI), Vol. 9271. Springer, 119-140.

Gervasio A. Lamas, J. David Knight, Michael O. Sweeney, Marcus Mianulli, Vinod Jorapur, Koroush Khalighi, James R. Cook, Russell Silverman, Lawrence Rosenthal, Nancy Clapp-Channing, Kerry L. Lee, and Daniel B. Mark. 2007. Impact of ratemodulated pacing on quality of life and exercise capacity evidence from the advanced elements of pacing randomized controlled trial (ADEPT). Heart Rhythm 4, 9 (2007), 1125-1132.

Chu-Pak Lau, Hung-Fat Tse, A. John Camm, and Serge S. Barold. 2007. Evolution of pacing for bradycardias: Sensors. European Heart fournal Supplements 9, suppl I (2007), I11-I22.

Jie Lian, Hannes Krätschmer, Dirk Müssig, and L. Stotts. 2010. Open source modeling of heart rhythm and cardiac pacing. Open Pacing Electrophysiology Therapy Journal 3 (2010), 4

Jie Lian, D. Mussig, and Volker Lang. 2006. Computer modeling of ventricular rhythm during atrial fibrillation and ventricular pacing. IEEE Transactions on Biomedical Engineering 53, 8 (2006), 1512-1520.

Hugo Daniel Macedo, Peter Gorm Larsen, and John Fitzgerald. 2008. Incremental development of a distributed real-time model of a cardiac pacing system using VDM. In Formal Methods. 181.

Anthony R. Magnano, Steve Holleran, Rajasekhar Ramakrishnan, James A. Reiffel, and Daniel M. Bloomfield. 2002. Autonomic nervous system influences on QT interval in normal subjects. Fournal of the American College of Cardiology 39, 11 (2002), 1820-1826.

Merak Malik, P. Färbom, V. Batchvarov, K. Hnatkova, and A. J. Camm. 2002. Relation between QT and RR intervals is highly individual among healthy subjects: Implications for heart rate correction of the QT interval. Heart 87, 3 (2002), $220-228$.

Jaakko Malmivuo and Robert Plonsey. 1995. Bioelectromagnetism: Principles and Applications of Bioelectric and Biomagnetic Fields. Oxford University Press.

Vias Markides and Richard J. Schilling. 2003. Atrial fibrillation: Classification, pathophysiology, mechanisms and drug treatment. Heart 89, 8 (2003), 939-943.

Patrick E. McSharry, Gari D. Clifford, Lionel Tarassenko, and Leonard A. Smith. 2003. A dynamical model for generating synthetic electrocardiogram signals. IEEE Transactions on Biomedical Engineering 50, 3 (2003), 289-294.

Dominique Méry, Bernhard Schätz, and Alan Wassyng. 2014. The pacemaker challenge: Developing certifiable medical devices (Dagstuhl seminar 14062). Dagstuhl Reports 4, 2 (2014), 17-37.

Dominique Méry and Neeraj Kumar Singh. 2009. Pacemaker's Functional Behaviors in Event-B. Rapport de recherche. Retrieved from http://hal.inria.fr/inria-00419973.

Benjamin Monteil, Sylvain Ploux, Romain Eschalier, Philippe Ritter, Michel Haissaguerre, Jayanthi N. Koneru, Kenneth A. Ellenbogen, and Pierre Bordachar. 2015. Pacemaker-mediated tachycardia: Manufacturer specifics and spectrum of cases. Pacing and Clinical Electrophysiology 38, 12 (2015), 1489-1498.

Kazunori Ohkawara, Yoshitake Oshima, Yuki Hikihara, Kazuko Ishikawa-Takata, Izumi Tabata, and Shigeho Tanaka. 2011. Real-time estimation of daily physical activity intensity by a triaxial accelerometer and a gravity-removal classification algorithm. British fournal of Nutrition 105 (6 2011), 1681-1691.

Miroslav Pajic, Zhihao Jiang, Insup Lee, Oleg Sokolsky, and Rahul Mangharam. 2012. From verification to implementation: A model translation tool and a pacemaker case study. In 2012 IEEE 18th Real-Time and Embedded Technology and Applications Symposium (RTAS'12). IEEE, 173-184.

Jiapu Pan and Willis J. Tompkins. 1985. A real-time QRS detection algorithm. IEEE Transactions on Biomedical Engineering 3 (1985), 230-236.

John W. Poore and Brian M. Mann. 1991. Self-adjusting rate-responsive pacemaker and method thereof. Retrieved from https://www.google.co.uk/patents/US5074302. US Patent 5,074,302.

Reza Sameni, Mohammad B. Shamsollahi, Christian Jutten, and Gari D. Clifford. 2007. A nonlinear bayesian filtering framework for ECG denoising. IEEE Transactions on Biomedical Engineering 54, 12 (2007), 2172-2185.

Dominic P. Searson. 2015. GPTIPS 2: An open-source software platform for symbolic data mining. In Handbook of Genetic Programming Applications. Springer.

Jae-Woo Shin, Jung-Han Yoon, and Y. R. Yoon. 2001. Rate-adaptive pacemaker controlled by motion and respiratory rate using neuro-fuzzy algorithm. Medical and Biological Engineering and Computing 39, 6 (2001), 694-699.

Hirofumi Tanaka, Kevin D. Monahan, and Douglas R. Seals. 2001. Age-predicted maximal heart rate revisited. fournal of the American College of Cardiology 37, 1 (2001), 153-156. 
Luu Anh Tuan, Man Chun Zheng, and Quan Thanh Tho. 2010. Modeling and verification of safety critical systems: A case study on pacemaker. In 2010 th International Conference on Secure Software Integration and Reliability Improvement (SSIRI'10). IEEE, 23-32.

Jin Wang, Ronghua Chen, Xiangping Sun, Mary F. H. She, and Yuchuan Wu. 2011. Recognizing human daily activities from accelerometer signal. Procedia Engineering 15 (2011), 1780-1786.

Bruce L. Wilkoff, Joseph Corey, and Gordon Blackburn. 1989. A mathematical model of the cardiac chronotropic response to exercise. fournal of Electrophysiology 3, 3 (1989), 176-180.

Pei Ye, Emilia Entcheva, Radu Grosu, and Scott A. Smolka. 2005. Efficient modeling of excitable cells using hybrid automata. In Proceedings of the 3rd International Conference on Computational Methods in Systems Biology, Vol. 5. 216-227.

Håkan L. S. Younes, Marta Kwiatkowska, Gethin Norman, and David Parker. 2006. Numerical vs. statistical probabilistic model checking. International fournal on Software Tools for Technology Transfer 8, 3 (2006), 216-228.

Received September 2016; revised May 2017; accepted October 2017 